TAIWANESE JOURNAL OF MATHEMATICS

Vol. 10, No. 3, pp. 739-775, March 2006

This paper is available online at http://www.math.nthu.edu.tw/tjm/

\title{
DISTRIBUTION COSINE FUNCTIONS
}

\author{
Marko Kostic
}

\begin{abstract}
A class of distribution cosine functions is introduced and systematically analyzed. Connections with (local) integrated cosine functions as well as some characterizations and related examples are given.
\end{abstract}

\section{INTRODUCTION}

It is well known that the notion of integrated cosine functions for a positive integral number $n$ was introduced by Arendt and Kellermann [2] in 1989. Then, the basic properties of exponentially bounded $\alpha$-times integrated cosine functions $(\alpha \geq 0)$ are investigated by many authors (cf., e.g., $[4,6,19,21]$ and references therein). Strongly continuous integrated $C$-cosine functions which are not necessarily exponentially bounded are investigated in [18]. The properties of $\mathcal{M}, \mathcal{N}$ functions, which in particular give the properties of cosine and sine functions, are given in [14] as well as the treatment of the second order abstract Cauchy problems (see also [20]). Connections of exponentially bounded integrated cosine functions with the abstract Cauchy problem $\left(A C P_{n}\right)$ are given in [19]. Perturbation and adjoint type results for $\alpha$-times integrated cosine functions are proved in [21].

On the other hand, distribution semigroups in Banach spaces and their generators were introduced by Lions in [13]. A new definition of distribution semigroups, covering in particular non-densely defined generators, is presented by Kunstmann in [9]. He proved that every generator of a distribution semigroup is also the generator of a global regularized semigroup and that a closed linear operator $A$ generates a distribution semigroup if and only if it is the generator of a local integrated semigroup. Similar results are independently obtained in [17], where Wang defined the same class of semigroups called by him quasi-distribution semigroups. Generator of

Received January 15, 2004, revised August 9, 2004.

Communicated by Sen-Yen Shaw.

2000 Mathematics Subject Classification: 47D03, 47D06, 47D60, 47D09.

Key words and phrases: Distribution cosine functions, Integrated cosine functions, Well-posedness. 
a degenerate distribution semigroup is introduced in [7], where some new characterizations of distribution semigroups are also shown. Basic theory of $C$-distribution semigroups is given in [8].

This paper is devoted to the study of distribution cosine functions and their generators (which are not necessarily densely defined). The theory obtained here can be viewed as a unification of the concept of (local) integrated cosine functions. Our investigations are mostly based on the theory of distribution semigroups. A straightforward approach seems to be more complicated.

The paper is organized as follows. In Section 1, we repeat some known facts related to integrated cosine functions and distribution semigroups. In this section we will prove Proposition 1.3 which clarify the relations between integrated cosine functions and integrated semigroups. This result seems to be new in local case. The notion of a distribution cosine function is introduced in Section 2. Afterwards we analyze the basic structural properties of distribution cosine functions. In Section 3 , we investigate the relations between distribution cosine functions and local integrated cosine functions, local $C$-cosine functions and convolution type equations. In Section 4 we introduce the notion of an exponential distribution cosine function and relate exponential distribution cosine functions to exponentially bounded integrated cosine functions. Section 5 is devoted to the study of dense distribution cosine functions. Last section gives several examples which justify our results given in previous sections.

\section{Preliminaries}

We use the standard notation. $E$ is a complex Banach space with the norm $\|\cdot\|$ and $L(E)=L(E, E)$ is the space of bounded linear operators from $E$ into $E$. We will assume that the space $E^{2}$ is topologized by the norm $\|(x, y)\|_{E^{2}}=\|x\|+\|y\|$.

By $\mathcal{D}$ we denote the space of all compactly supported $C^{\infty}$-functions from $\mathbb{R}$ into $\mathbb{C}$ equipped with the usual inductive limit topology; $\mathcal{E}$ is the space of all $C^{\infty}$ functions $\mathbb{R} \rightarrow \mathbb{C}$ supplied with the usual topology and $\mathcal{S}$ denotes the Schwartz space of all rapidly decreasing functions. Their strong duals are $\mathcal{D}^{\prime}, \mathcal{E}^{\prime}$ and $\mathcal{S}^{\prime}$, respectively. By $\mathcal{D}_{0}$ we denote the subspace of $\mathcal{D}$ which consists of the elements supported by $[0, \infty)$. Further on, $\mathcal{D}^{\prime}(L(E))=L(\mathcal{D}, L(E)), \mathcal{E}^{\prime}(L(E))=L(\mathcal{E}, L(E))$ and $\mathcal{S}^{\prime}(L(E))=L(\mathcal{S}, L(E))$ are spaces of continuous linear functions $\mathcal{D} \rightarrow L(E)$, $\mathcal{E} \rightarrow L(E)$ and $\mathcal{S} \rightarrow L(E)$, respectively, equipped with the topology of uniform convergence on bounded subsets of $\mathcal{D}, \mathcal{E}$ and $\mathcal{S}$, respectively; $\mathcal{D}_{0}^{\prime}(L(E)), \mathcal{E}_{0}^{\prime}(L(E))$ and $\mathcal{S}_{0}^{\prime}(L(E))$ are the subspaces of $\mathcal{D}^{\prime}(L(E)), \mathcal{E}^{\prime}\left((L(E))\right.$ and $\mathcal{S}^{\prime}((L(E))$, respectively, containing the elements supported by $[0, \infty)$.

For a linear operator $A$, its domain, range and null space are denoted by $D(A), R(A)$ and $N(A)$, respectively. We will always assume that $A$ is a closed 
operator and that $C \in L(E)$ is an injective operator; $[D(A)]$ denotes the Banach space $D(A)$ equipped with the graph norm. Let $K \subset \mathbb{R}$. Then $\mathcal{D}_{K}$ denotes the subspace of $\mathcal{D}$ which consists of the test functions whose support is contained in $K$. Let $\alpha \in \mathcal{D}_{[-2,-1]}$ be a fixed test function satisfying $\int_{-\infty}^{\infty} \alpha(x) d x=1$. Then, with $\alpha$ chosen in this way, for every fixed $\varphi$ we define $I(\varphi)$ as follows

$$
I(\varphi)(x):=\int_{-\infty}^{x}\left[\varphi(t)-\alpha(t) \int_{-\infty}^{\infty} \varphi(u) d u\right] d t, x \in \mathbb{R} .
$$

It is clear that $I(\varphi) \in \mathcal{D}, I\left(\varphi^{\prime}\right)=\varphi$ and $\frac{d}{d x} I(\varphi)(x)=\varphi(x)-\alpha(x) \int_{-\infty}^{\infty} \varphi(u) d u, x \in$ $\mathbb{R}$. Then, for $G \in \mathcal{D}^{\prime}(L(E))$ we may define $G^{-1}$ via $G^{-1}(\varphi):=-G(I(\varphi)), \varphi \in$ $\mathcal{D}$. We have $G^{-1} \in \mathcal{D}^{\prime}(L(E))$ and $\left(G^{-1}\right)^{\prime}=G$; more precisely, $-G^{-1}\left(\varphi^{\prime}\right)=$ $G\left(I\left(\varphi^{\prime}\right)\right)=G(\varphi), \varphi \in \mathcal{D}$.

Let $\varphi \in \mathcal{D}$ with $\operatorname{supp} \varphi \subset(-\infty, 0)$ be fixed. Our choice of $\alpha$ implies $\frac{d}{d x} I(\varphi)(x)=0, x>a$, for a suitable $a \in(-\infty, 0)$. Accordingly, $\operatorname{supp} I(\varphi) \subset$ $(-\infty, 0)$. It implies the following:

$\operatorname{supp} G \subset[0, \infty) \Rightarrow \operatorname{supp} G^{-1} \subset[0, \infty)$. Moreover, if $\varphi \in \mathcal{D}$ with $\operatorname{supp} \varphi \subset[a, b]$, then

$$
\operatorname{supp} I(\varphi) \subset[\min (-2, a), \max (-1, b)] .
$$

Let $k \in \mathbb{N}_{0}$. The distribution $\delta^{(k)}$ is defined by $\delta^{(k)}(\varphi)=(-1)^{k} \varphi^{(k)}(0), \varphi \in \mathcal{D}$.

For a $\varphi \in \mathcal{D}$ and an $f \in \mathcal{D}^{\prime}$, or for a $\varphi \in \mathcal{E}$ and an $f \in \mathcal{E}^{\prime}$, we define the convolution $f * \varphi$ by

$$
(f * \varphi)(t):=f(\varphi(t-\cdot)), t \in \mathbb{R} .
$$

For $f \in \mathcal{D}^{\prime}$, or for $f \in \mathcal{E}^{\prime}$, define $\check{f}$ by $\check{f}(\varphi):=f(\varphi(-)), \varphi \in \mathcal{D}(\varphi \in \mathcal{E})$. Generally, the convolution of two distribution $f, g \in \mathcal{D}^{\prime}, f * g \in \mathcal{D}^{\prime}$, is defined by $(f * g)(\varphi)=g(\check{f} * \varphi), \varphi \in \mathcal{D}$. We have $\operatorname{supp}(f * g) \subset \operatorname{supp} f+\operatorname{supp} g$. It is known that $f * \varphi \in \mathcal{D}_{0}$, if $f \in \mathcal{E}_{0}^{\prime}$ and $\varphi \in \mathcal{D}_{0}$.

We give some elementary facts concerning $\alpha$-times integrated cosine functions and distribution semigroups in the sense of [13] and [9]. We refer to [1], [3], [11], [12] and [19] for the material intimately related to (local) integrated $C$-semigroups.

Definition 1.1. Let $A$ be a closed operator, $\alpha \geq 0,0<\tau \leq \infty$. If there exists a strongly continuous operator family $\left(C_{\alpha}(t)\right)_{t \in[0, \tau)}\left(C_{\alpha}(t) \in L(E), 0 \leq t<\tau\right)$ such that:

(i) $C_{\alpha}(t) A \subset A C_{\alpha}(t), t \in[0, \tau)$, 
(ii) for all $x \in E$ and $t \in[0, \tau)$ :

$$
\begin{gathered}
\int_{0}^{t}(t-s) C_{\alpha}(s) x d s \in D(A), \text { and } \\
A \int_{0}^{t}(t-s) C_{\alpha}(s) x d s=C_{\alpha}(t) x-\frac{t^{\alpha}}{\Gamma(\alpha+1)} x,
\end{gathered}
$$

then $C_{\alpha}$ is called a (local) $\alpha$-times integrated cosine function and $A$ is called the generator of $C_{\alpha}$.

The following proposition can be easily proved (cf. also [21]) by standard arguments.

Proposition 1.2. Let $\left(C_{\alpha}(t)\right)_{t \geq 0}$ be a strongly continuous, exponentially bounded operator family and let $A$ be a closed operator. Then $A$ is the generator of the $\alpha$-times integrated cosine function $C_{\alpha}$ if and only if there exists $\omega>0$ such that $\left(\omega^{2}, \infty\right) \subset \rho(A)$ and

$$
\lambda R\left(\lambda^{2}: A\right) x=\lambda^{\alpha} \int_{0}^{\infty} e^{-\lambda t} C_{\alpha}(t) x d t, \lambda>\omega, x \in E .
$$

Now, Definition 1.1 leads us to the next question: if $\left(C_{\alpha}(t)\right)_{t \in[0, \tau)}$ is a (local) $\alpha$-times integrated cosine function, what is its 'semigroup' relation? In this paper it will be enough to give the answer when $\alpha=n \in \mathbb{N}$. Essentially, the answer is contained in [18] where the semigroup property of a global integrated $C$-cosine function is discussed. Repeating literally the arguments given in [18], one obtains:

$$
\begin{aligned}
2 C(t) C(s) x= & \frac{1}{(n-1) !}\left[(-1)^{n} \int_{0}^{|t-s|}(|t-s|-r)^{n-1} C(r) x d r\right. \\
& +\left[\int_{0}^{t+s}-\int_{0}^{t}-\int_{0}^{s}\right](t+s-r)^{n-1} C(r) x d r \\
& +\int_{0}^{t}(s-t+r)^{n-1} C(r) x d r \\
& \left.+\int_{0}^{s}(t-s+r)^{n-1} C(r) x d r\right], 0 \leq t, s, t+s<\tau, x \in E .
\end{aligned}
$$

All this has been seen many times and the details are omitted here. So, if $\left(C_{n}(t)\right)_{t \in[0, \tau)}$ is an $n$-times integrated cosine function then $C_{n}(t) C_{n}(s)=C_{n}(s) C_{n}$ 
$(t), 0 \leq t, s<\frac{\tau}{2}$. This equality remains true for all $(t, s) \in[0, \tau) \times[0, \tau)$; it will be proved soon. We refer to [21] for the 'semigroup' relation in the case $\alpha \geq 0$.

Now we give an important result for our further investigations. Its exponential version is well known (see for instance [21]).

Proposition 1.3. Let $A$ be a closed operator, $\alpha \geq 0,0<\tau \leq \infty$. Then the following assertions are equivalent:

(i) $A$ is the generator of an $\alpha$-times integrated cosine function $\left(C_{\alpha}(t)\right)_{t \in[0, \tau)}$ in E.

(ii) The operator $\mathcal{A} \equiv\left(\begin{array}{ll}0 & I \\ A & 0\end{array}\right)$ is the generator of an $(\alpha+1)$-times integrated semigroup $\left(S_{\alpha+1}(t)\right)_{t \in[0, \tau)}$ in $E^{2}$.

In this case, we have

$$
S_{\alpha+1}(t)=\left(\begin{array}{cc}
\int_{0}^{t} C_{\alpha}(s) d s & \int_{0}^{t}(t-s) C_{\alpha}(s) d s \\
C_{\alpha}(t)-\frac{t^{\alpha}}{\Gamma(\alpha+1)} I & \int_{0}^{t} C_{\alpha}(s) d s
\end{array}\right), 0 \leq t<\tau .
$$

Proof. (i) $\Rightarrow$ (ii). One obtains that $\left(S_{\alpha+1}(t)\right)_{t \in[0, \tau)}$ is a strongly continuous operator family in $E^{2}$ and that $S_{\alpha+1}(t) \mathcal{A} \subset \mathcal{A} S_{\alpha+1}(t), 0 \leq t<\tau$. Moreover,

$$
\begin{aligned}
\mathcal{A} \int_{0}^{t} S_{\alpha+1}(s)\left(\begin{array}{l}
x \\
y
\end{array}\right) d s & =\mathcal{A} \int_{0}^{t}\left(\begin{array}{l}
\int_{0}^{s} C_{\alpha}(r) x d r+\int_{0}^{s}(s-r) C_{\alpha}(r) y d r \\
C_{\alpha}(s) x-\frac{s^{\alpha}}{\Gamma(\alpha+1)} x+\int_{0}^{s} C_{\alpha}(r) y d r
\end{array}\right) d s \\
& =\mathcal{A}\left(\begin{array}{l}
\int_{0}^{t}(t-s) C_{\alpha}(s) x d s+\int_{0}^{t} \frac{(t-s)^{2}}{2} C_{\alpha}(s) y d s \\
\int_{0}^{t} C_{\alpha}(s) x d s-\frac{t^{\alpha+1}}{\Gamma(\alpha+2)} x+\int_{0}^{t}(t-s) C_{\alpha}(s) y d s
\end{array}\right) \\
& =\left(\begin{array}{l}
\int_{0}^{t} C_{\alpha}(s) x d s-\frac{t^{\alpha+1}}{\Gamma(\alpha+2)} x+\int_{0}^{t}(t-s) C_{\alpha}(s) y d s \\
C_{\alpha}(t) x-\frac{t^{\alpha}}{\Gamma(\alpha+1)} x+\int_{0}^{t} C_{\alpha}(s) y d s-\frac{t^{\alpha+1}}{\Gamma(\alpha+2)} y
\end{array}\right) \\
& =S_{\alpha+1}(t)\left(\begin{array}{l}
x \\
y
\end{array}\right)-\frac{t^{\alpha+1}}{\Gamma(\alpha+2)}\left(\begin{array}{l}
x \\
y
\end{array}\right), 0 \leq t<\tau, x, y \in E
\end{aligned}
$$


(ii) $\Rightarrow$ (i). Let $S_{\alpha+1}(t)=\left(\begin{array}{ll}S_{\alpha+1}^{1}(t) & S_{\alpha+1}^{2}(t) \\ S_{\alpha+1}^{3}(t) & S_{\alpha+1}^{4}(t)\end{array}\right)_{t \in[0, \tau)}$ and $S_{\alpha+1}^{i}(t) \in L(E), i \in\{1,2,3,4\}, 0 \leq t<\tau$. Since $S_{\alpha+1} \mathcal{A} \subset \mathcal{A} S_{\alpha+1}$, we obtain

$$
\begin{gathered}
S_{\alpha+1}^{1}(t) x+S_{\alpha+1}^{2}(t) y \in D(A), \\
S_{\alpha+1}^{1}(t) y+S_{\alpha+1}^{2}(t) A x=S_{\alpha+1}^{3}(t) x+S_{\alpha+1}^{4}(t) y, \\
S_{\alpha+1}^{3}(t) y+S_{\alpha+1}^{4}(t) A x=A\left(S_{\alpha+1}^{1}(t) x+S_{\alpha+1}^{2}(t) y\right), 0 \leq t<\tau, x \in D(A), y \in E .
\end{gathered}
$$

Hence, $S_{\alpha+1}^{3}(t) x=S_{\alpha+1}^{2}(t) A x, x \in D(A)$, and $S_{\alpha+1}^{3}(t) y=A S_{\alpha+1}^{2}(t) y, y \in E$, $0 \leq t<\tau$. This implies that for every $x \in D(A)$, we have $S_{\alpha+1}^{3}(t) A x=$ $A S_{\alpha+1}^{2}(t) A x=A S_{\alpha+1}^{3}(t) x$. Thus, $S_{\alpha+1}^{3}(t) A \subset A S_{\alpha+1}^{3}(t), t \in[0, \tau)$, and $\left(S_{\alpha+1}^{3}+\right.$ $\left.\frac{t^{\alpha}}{\Gamma(\alpha+1)} I\right)_{t \in[0, \tau)}$ is a strongly continuous operator family. Now, the simple calculation deduced from

$$
\mathcal{A} \int_{0}^{t} S_{\alpha+1}(s)\left(\begin{array}{l}
x \\
y
\end{array}\right) d s=S_{\alpha+1}(t)\left(\begin{array}{l}
x \\
y
\end{array}\right)-\frac{t^{\alpha+1}}{\Gamma(\alpha+2)}\left(\begin{array}{l}
x \\
y
\end{array}\right),
$$

gives

$$
\int_{0}^{t} S_{\alpha+1}^{3}(s) x d s+\int_{0}^{t} S_{\alpha+1}^{4}(s) y d s=S_{\alpha+1}^{1}(t) x+S_{\alpha+1}^{2}(t) y-\frac{t^{\alpha+1}}{\Gamma(\alpha+2)} x,
$$

and

$$
\begin{aligned}
& A\left[\int_{0}^{t} S_{\alpha+1}^{1}(s) x d s+\int_{0}^{t} S_{\alpha+1}^{2}(s) y d s\right] \\
= & S_{\alpha+1}^{3}(t) x+S_{\alpha+1}^{4}(t) y-\frac{t^{\alpha+1}}{\Gamma(\alpha+2)} y, 0 \leq t<\tau, x, y \in E .
\end{aligned}
$$

Thus, $\int_{0}^{t} S_{\alpha+1}^{3}(s) x d s=S_{\alpha+1}^{1}(t) x-\frac{t^{\alpha+1}}{\Gamma(\alpha+2)} x$, and $A \int_{0}^{t} S_{\alpha+1}^{1}(s) x d s=S_{\alpha+1}^{3}(t) x$, $0 \leq t<\tau, x \in E$.

Consequently,

$$
\begin{aligned}
& A\left[\int_{0}^{t}(t-s)\left(S_{\alpha+1}^{3}(s) x+\frac{s^{\alpha}}{\Gamma(\alpha+1)} x\right) d s\right] \\
= & A\left[\int_{0}^{t}(t-s)\left(\frac{d}{d v} S_{\alpha+1}^{1}(v) x\right)_{v=s} d s\right]=A \int_{0}^{t} S_{\alpha+1}^{1}(s) x d s
\end{aligned}
$$




$$
=\left[S_{\alpha+1}^{3}(t) x+\frac{t^{\alpha}}{\Gamma(\alpha+1)} x\right]-\frac{t^{\alpha}}{\Gamma(\alpha+1)} x, 0 \leq t<\tau, x \in E, \text { and }
$$

$A$ is the generator of the $\alpha$-times integrated cosine function $\left(S_{\alpha+1}^{3}(t)+\frac{t^{\alpha}}{\Gamma(\alpha+1)} I\right)_{t \in[0, \tau)}$. Clearly, $S_{\alpha+1}^{1}(t)=S_{\alpha+1}^{4}(t)$ and $S_{\alpha+1}^{2}(t)=\int_{0}^{t} S_{\alpha+1}^{1}(s) d s, 0 \leq t<\tau$.

Note, if $\tau=\infty$, then $C_{\alpha}$ is exponentially bounded if and only if $S_{\alpha+1}$ is exponentially bounded.

Remark 1.4. Every $\alpha$-times integrated cosine function $\left(C_{\alpha}(t)\right)_{t \in[0, \tau)}(\alpha \geq 0)$ is uniquely determined by its generator $A$. This fact follows from Proposition 1.3 and the corresponding statement in the case of (local) integrated semigroups.

Now we are able to prove the next proposition.

Proposition 1.5. Let $A$ be the generator of an n-times integrated cosine function $\left(C_{n}(t)\right)_{t \in[0, \tau)}, n \in \mathbb{N}_{0}$. Then $A$ generates a $(2 n+1)$-times integrated cosine function $\left(C_{2 n+1}(t)\right)_{t \in[0,2 \tau)}$ and $C_{n}(t) C_{n}(s)=C_{n}(s) C_{n}(t), 0 \leq t, s<\tau$.

Proof. It follows from the assumption that the operator $\mathcal{A}$ is the generator of an $(n+1)$-times integrated semigroup $\left(S_{n+1}(t)\right)_{t \in[0, \tau)}$. By [1, Theorem 4.1], $\mathcal{A}$ generates a $(2 n+2)$-times integrated semigroup $\left(S_{2 n+2}(t)\right)_{t \in[0,2 \tau)}$. Hence, $A$ generates a $(2 n+1)$-times integrated cosine function $\left(C_{2 n+1}(t)\right)_{t \in[0,2 \tau)}$ satisfying $C_{2 n+1}(t) C_{2 n+1}(s)=C_{2 n+1}(s) C_{2 n+1}(t), 0 \leq t, s<\tau$. The previous remark implies $C_{2 n+1}(t)=\int_{0}^{t} \frac{(t-u)^{n}}{n !} C_{n}(u) d u, 0 \leq t<\tau$, and therefore

$$
\begin{gathered}
\frac{d^{n+1}}{d t^{n+1}} \frac{d^{n+1}}{d s^{n+1}}\left[\int_{0}^{t} \frac{(t-u)^{n}}{n !} C_{n}(u) x d u \int_{0}^{s} \frac{(s-u)^{n}}{n !} C_{n}(u) x d u\right] \\
=\frac{d^{n+1}}{d t^{n+1}} \frac{d^{n+1}}{d s^{n+1}}\left[\int_{0}^{s} \frac{(s-u)^{n}}{n !} C_{n}(u) x d u \int_{0}^{t} \frac{(t-u)^{n}}{n !} C_{n}(u) x d u\right], 0 \leq t, s<\tau, x \in E .
\end{gathered}
$$

Accordingly, $C_{n}(t) C_{n}(s)=C_{n}(s) C_{n}(t), 0 \leq t, s<\tau$.

Next we give the definitions of distribution semigroups in the sense of [13] and [9].

Definition 1.6. (Lions, [13]). An $L$-distribution semigroup, (L-DSG) in short, 
is an element $G \in \mathcal{D}_{0}^{\prime}(L(E))$ satisfying

$$
\begin{aligned}
&\left(d_{1}\right): G(\varphi * \psi)=G(\varphi) G(\psi), \varphi, \psi \in \mathcal{D}_{0} \\
&\left(d_{2}\right): \mathcal{N}(G):=\bigcap_{\varphi \in \mathcal{D}_{0}} \operatorname{Kern} G(\varphi)=\{0\} \\
&\left(d_{3}\right): \mathcal{R}(G):=\bigcup_{\varphi \in \mathcal{D}_{0}} \operatorname{Im} G(\varphi) \text { is dense in } E \\
&\left(d_{4}\right): \text { for all } x \in \mathcal{R}(G) \text { there exists } u \in C([0, \infty): E) \text { satisfying } \\
& \\
& \quad u(0)=x \text { and } G(\psi) x=\int_{0}^{\infty} u(t) \psi(t) d t, \psi \in \mathcal{D} .
\end{aligned}
$$

Definition 1.7. (Kunstmann, [9]). An element $G \in \mathcal{D}_{0}^{\prime}(L(E))$ is called a pre-(DSG) if it satisfies

$$
\left(q d_{1}\right): \quad G\left(\varphi *_{0} \psi\right)=G(\varphi) G(\psi), \quad \varphi, \psi \in \mathcal{D},
$$

where $\varphi *_{0} \psi(t):=\int_{0}^{t} \varphi(t-s) \psi(s) d s, t \in \mathbb{R}$, and it is called a distribution semigroup, (DSG) shortly, if it additionally satisfies $\left(d_{2}\right)$. Moreover, if $G$ satisfies $\left(d_{3}\right)$ then $G$ is called a dense (DSG).

Obviously, if $G$ is an (L-DSG), then it is a (DSG). The converse is true if and only if $G$ is a dense (DSG).

Let $G \in \mathcal{D}_{0}^{\prime}(L(E))$ satisfy $\left(d_{1}\right),\left(d_{2}\right)$ and let $T \in \mathcal{E}_{0}^{\prime}$. Define $G(T)$ on a subspace of $E$ by

$$
y=G(T) x \text { if and only if } G(T * \varphi) x=G(\varphi) y \text { for all } \varphi \in \mathcal{D}_{0} .
$$

Denote its domain by $D(G(T))$. Linearity and closedness of the operator $G(T)$ : $D(G(T)) \rightarrow E$ are obvious.

The generator of a (DSG) $G$ is defined by $A:=G\left(-\delta^{\prime}\right)$; this definition can be used for the generator of an (L-DSG). Since for $\varphi \in \mathcal{D}, \varphi_{+}:=\varphi 1_{[0, \infty)} \in \mathcal{E}_{0}^{\prime}$, $\left(1_{[0, \infty)}\right.$ is the characteristic function of $\left.[0, \infty)\right)$ it is clear what $G\left(\varphi_{+}\right)$means.

Proposition 1.8. Let $G \in \mathcal{D}_{0}^{\prime}(L(E))$. Then $G$ is a (DSG) if and only if $\left(d_{1}\right)$, $\left(d_{2}\right)$ and $\left(d_{5}\right)$ hold, where

$$
\left(d_{5}\right): G\left(\varphi_{+}\right)=G(\varphi), \varphi \in \mathcal{D} .
$$

Proof. Suppose that $G$ is a (DSG). It is known that $G(T)$ commutes with $G(\varphi)$ for all $T \in \mathcal{E}_{0}^{\prime}$ and $\varphi \in \mathcal{D}$ ([9, Lemma 3.6(b)]). Moreover, the set $\mathcal{R}(G)$ is contained 
in $D(G(T))$. Let $x \in E$. Since for every $\psi \in \mathcal{D}$ and $\varphi \in \mathcal{D}_{0}: \varphi *_{0} \psi=\varphi * \psi_{+}$, we have $G\left(\psi_{+} * \varphi\right) x=G\left(\varphi *_{0} \psi\right) x=G(\varphi) G(\psi) x$ and $\left(d_{5}\right)$ holds. Suppose conversely that $G \in \mathcal{D}_{0}^{\prime}(L(E))$ satisfies $\left(d_{1}\right),\left(d_{2}\right)$ and $\left(d_{5}\right)$. We have to prove $\left(q d_{1}\right)$. Firstly, let us assume that $S, T \in \mathcal{E}_{0}^{\prime}$. Since $G(\cdot)$ satisfies $\left(d_{1}\right)$ and $\left(d_{2}\right)$, it is clear that $G(S)$ is a closed linear operator. We prove that $G(S) G(T) \subset G(S * T)$ and that $D(G(S) G(T))=D(G(S * T)) \cap D(G(T))$ (cf. also [9, Lemma 3.6]). Let $x \in D(G(S) G(T))$. Then $x \in D(G(T))$ and $y:=G(T) x \in D(G(S))$. Furthermore, for every $x \in E$ and $\varphi \in \mathcal{D}_{0}, G(T * \varphi) x=G(\varphi) y=G(\varphi) G(T) x$ and

$$
G(S * T * \varphi) x=G(T * S * \varphi) x=G(S * \varphi) G(T) x=G(\varphi) G(S) G(T) x .
$$

It implies that $x \in D(G(S * T)) \cap D(G(T))$ and that $G(S) G(T) \subset G(S * T)$. Assume that $x \in D(G(S * T)) \cap D(G(T))$. We prove that $G(T) x \in D(G(S))$ and, consequently, $x \in D(G(S) G(T))$. Let $\varphi \in \mathcal{D}_{0}$. Then the definition of $G(T)$ implies

$G(S * \varphi) G(T) x=G(T *(S * \varphi)) x=G((S * T) * \varphi) x=G(\varphi) G(S * T) x, \varphi \in \mathcal{D}_{0}$, which gives $G(T) x \in D(G(S))$ and $G(S) G(T) x=G(S * T) x$.

Let $\varphi, \psi \in \mathcal{D}$. Then $\left(\varphi *_{0} \psi\right)_{+}=\varphi_{+} * \psi_{+}$. By $\left(d_{5}\right)$, one obtains $G\left(\varphi *_{0} \psi\right)=$ $G\left(\varphi_{+} * \psi_{+}\right)=G\left(\varphi_{+}\right) G\left(\psi_{+}\right)=G(\varphi) G(\psi)$ and the proof is now completed.

Lemma 1.9. Suppose that $G$ is a (DSG). Then $G$ satisfies $\left(d_{4}\right)$.

Proof. The proof is essentially given on page 846 of [9]; see [8] for a more general result. If $f: \mathbb{R} \rightarrow \mathbb{C}$, let $\left(\tau_{t} f\right)(s)=f(s-t), s \in \mathbb{R}, t \in \mathbb{R}$. Suppose that $x=G(\varphi) y$ for some $\varphi \in \mathcal{D}_{0}$ and $y \in E$. By the continuity of $G$ on $\mathcal{D}$, we obtain for every $\psi \in \mathcal{D}$ :

$G(\psi) x=G(\psi) G(\varphi) y=G\left(\psi *_{0} \varphi\right) y=G\left(\int_{0}^{\infty} \psi(t) \tau_{t} \varphi d t\right) y=\int_{0}^{\infty} \psi(t) G\left(\tau_{t} \varphi\right) y d t$.

Put $u(t)=G\left(\tau_{t} \varphi\right) y, t \geq 0$. Then $u$ has the desired properties.

For the convenience of a reader, we repeat the construction given in the proofs of [1, Theorem 7.2] and [17, Theorem 3.8]. Let $\left(S_{n}(t)\right)_{t \in[0, \tau)}$ be a local $n$-times integrated semigroup generated by $A$. Then it is proved in [1, Theorem 4.1] that $A$ generates a local $2 n$-times integrated semigroup on $[0,2 \tau)$. By induction, for every $k \in \mathbb{N}, A$ generates a local $2^{k} n$-times integrated semigroup $\left(S_{2^{k} n}(t)\right)_{t \in\left[0,2^{k} \tau\right)}$. Let $\varphi \in \mathcal{D}_{\left(-\infty, 2^{k} \tau\right)}$ and $x \in E$. Define $G(\varphi) x:=(-1)^{2^{k} n} \int_{0}^{2^{k} \tau} \varphi^{\left(2^{k} n\right)}(t) S_{2^{k} n}(t) x d t$. It is proved in [17, Theorem 3.8] that $G$ is a (DSG) generated by $A$. 


\section{Lemma 1.10.}

(a) Let $A$ be a closed linear operator and $\lambda \in \mathbb{C}$. Then $\lambda \in \rho(\mathcal{A}) \Leftrightarrow \lambda^{2} \in \rho(A)$. In this case, $\|R(\lambda: \mathcal{A})\| \leq(1+|\lambda|) \sqrt{1+|\lambda|^{2}}\left\|R\left(\lambda^{2}: A\right)\right\|+1$, and $\left\|R\left(\lambda^{2}: A\right)\right\| \leq\|R(\lambda: \mathcal{A})\|$.

(b) Let $\emptyset \neq \Omega \subset \mathbb{C}$. Put $\Omega^{2}:=\left\{\lambda^{2}: \lambda \in \Omega\right\}$. Then $\Omega \subset \rho(\mathcal{A})$ if and only if $\Omega^{2} \subset \rho(A)$; if this is the case, then $\|R(\cdot: A)\|$ is polynomially bounded on $\Omega^{2}$ if and only if $\|R(\cdot: \mathcal{A})\|$ is polynomially bounded on $\Omega$.

Proof. Suppose that $\lambda^{2} \in \rho(A)$. Then $\lambda \in \rho(\mathcal{A})$ and

$$
R(\lambda: \mathcal{A})\left(\begin{array}{l}
x \\
y
\end{array}\right)=\left(\begin{array}{c}
R\left(\lambda^{2}: A\right)(\lambda x+y) \\
A R\left(\lambda^{2}: A\right) x+\lambda R\left(\lambda^{2}: A\right) y
\end{array}\right), x, y \in E .
$$

See also [3, p. 212]. Let $x, y \in E$. We have

$$
\begin{aligned}
\left\|R(\lambda: \mathcal{A})\left(\begin{array}{l}
x \\
y
\end{array}\right)\right\|= & \left\|R\left(\lambda^{2}: A\right)(\lambda x+y)\right\|+\left\|\lambda^{2} R\left(\lambda^{2}: A\right) x-x+\lambda R\left(\lambda^{2}: A\right) y\right\| \\
= & \left\|R\left(\lambda^{2}: A\right)(\lambda x+y)\right\|+\left\|\left(\lambda^{2} R\left(\lambda^{2}: A\right) x+\lambda R\left(\lambda^{2}: A\right) y\right)-x\right\| \\
\leq & (1+|\lambda|)\left\|R\left(\lambda^{2}: A\right)(\lambda x+y)\right\|+\|x\| \leq(1+|\lambda|)\left\|R\left(\lambda^{2}: A\right)\right\| \\
& (|\lambda|\|x\|+\|y\|)+\|x\| \\
\leq & \left((1+|\lambda|) \sqrt{1+|\lambda|^{2}}\left\|R\left(\lambda^{2}: A\right)\right\|+1\right)\left\|\left(\begin{array}{l}
x \\
y
\end{array}\right)\right\| .
\end{aligned}
$$

Assume now $\lambda \in \rho(\mathcal{A})$. Let us show that $R\left(\lambda^{2} I-A\right)=E$. Let $x \in E$ be fixed. Put $\left(\begin{array}{l}u \\ v\end{array}\right)=R(\lambda: \mathcal{A})\left(\begin{array}{l}0 \\ x\end{array}\right)$. Then

$$
\left(\begin{array}{cc}
\lambda I & -I \\
-A & \lambda I
\end{array}\right)\left(\begin{array}{l}
u \\
v
\end{array}\right)=\left(\begin{array}{l}
0 \\
x
\end{array}\right)
$$

and it implies $\lambda u-v=0$ and $\lambda v-A u=x$. Therefore, $\lambda^{2} u-\lambda v=0$ and $\lambda^{2} u-A u=x$. We show that the operator $\lambda^{2} I-A$ is injective. Suppose that $\lambda^{2} x-A x=0$, for some $x \in E$. Then

$$
\left(\begin{array}{cc}
\lambda I & -I \\
-A & \lambda I
\end{array}\right)\left(\begin{array}{c}
x \\
\lambda x
\end{array}\right)=\left(\begin{array}{l}
0 \\
0
\end{array}\right)
$$

Consequently, $\left(\begin{array}{c}x \\ \lambda x\end{array}\right)=R(\lambda: \mathcal{A})\left(\begin{array}{l}0 \\ 0\end{array}\right)=\left(\begin{array}{l}0 \\ 0\end{array}\right)$, and $x=0$.

Since $\lambda^{2} I-A$ is closed, it follows from the above arguments and the closed graph theorem that $\left(\lambda^{2} I-A\right)^{-1}$ is bounded, i.e., $\lambda^{2} \in \rho(A)$. From the above given arguments, we have $\left\|R\left(\lambda^{2}: A\right)\right\| \leq\|R(\lambda: \mathcal{A})\|$. This proves (a). The proof of (b) follows instantly from that of (a). 
2. Elementary Properties of Distribution Cosine Functions

Definition 2.1. An element $G \in \mathcal{D}_{0}^{\prime}(L(E))$ is called a pre-(DCF) if it satisfies

$$
\left(D C F_{1}\right): \quad G^{-1}\left(\varphi *_{0} \psi\right)=G^{-1}(\varphi) G(\psi)+G(\varphi) G^{-1}(\psi), \varphi, \psi \in \mathcal{D},
$$

and it is called a distribution cosine function, in short (DCF), if it additionally satisfies

$\left(D C F_{2}\right): \quad x=y=0$ if and only if $G(\varphi) x+G^{-1}(\varphi) y=0$, for all $\varphi \in \mathcal{D}_{0}$.

A pre-(DCF) $G$ is dense if $\mathcal{R}(G):=\bigcup_{\varphi \in \mathcal{D}_{0}} \operatorname{Im} G(\varphi)$ is dense in $E$.

$\left(D C F_{2}\right)$ implies $\bigcap_{\varphi \in \mathcal{D}_{0}} \operatorname{Kern} G(\varphi)=\{0\}$ and $\bigcap_{\varphi \in \mathcal{D}_{0}} \operatorname{Kern}^{-1}(\varphi)=\{0\}$. From Definition 2.1, it is also clear that if $G$ is a pre-(DCF), then $G \in \mathcal{D}_{0}^{\prime}(L(E))$ and hence $G(\varphi)=0$ for all $\varphi \in \mathcal{D}_{(-\infty, 0]}$. It is not clear whether the condition $\left(\bigcap_{\varphi \in \mathcal{D}_{0}} \operatorname{Kern} G(\varphi) \supset\right) \bigcap_{\varphi \in \mathcal{D}_{0}} \operatorname{Kern} G^{-1}(\varphi)=\{0\}$ implies $\left(D C F_{2}\right)$.

Someone may think that $\left(D C F_{2}\right)$ is a crude assumption. But, this is a right 'nondegenerate' condition as the next proposition shows.

Proposition 2.2. Let $G \in \mathcal{D}_{0}^{\prime}(L(E))$. Then $G$ is a pre-(DCF) in $E$ if and only if

$\mathcal{G} \equiv\left(\begin{array}{cc}G & G^{-1} \\ G^{\prime}-\delta & G\end{array}\right)$ is a pre-(DSG) in $E^{2}$. Moreover, $\mathcal{G}$ is a (DSG) if and only if $G$ is a pre-(DCF) which satisfies $\left(D C F_{2}\right)$.

Proof. Since $\alpha \in \mathcal{D}_{[-2,-1]}$, we have $\mathcal{G} \in \mathcal{D}_{0}^{\prime}\left(L\left(E^{2}\right)\right)$. The simple calculation shows that $\mathcal{G}$ satisfies $\left(q d_{1}\right)$ if and only if the following holds:

(i) $G^{-1}\left(\varphi *_{0} \psi\right)=G^{-1}(\varphi) G(\psi)+G(\varphi) G^{-1}(\psi)$,

(ii) $G\left(\varphi *_{0} \psi\right)=G(\varphi) G(\psi)+G^{-1}(\varphi)\left(G^{\prime}-\delta\right)(\psi)$,

(iii) $G^{\prime}\left(\varphi *_{0} \psi\right)=\left(G^{\prime}-\delta\right)(\varphi) G(\psi)+G(\varphi)\left(G^{\prime}-\delta\right)(\psi), \varphi, \psi \in \mathcal{D}$.

We will show (i) $\Rightarrow$ (ii) $\Rightarrow$ (iii). Suppose (i) holds. Since $\left(\varphi *_{0} \psi\right)^{\prime}=\varphi^{\prime} *_{0} \psi+$ $\varphi(0) \psi=\varphi *_{0} \psi^{\prime}+\psi(0) \varphi, \varphi, \psi \in \mathcal{D}$, we have

$$
\begin{aligned}
G\left(\varphi *_{0} \psi\right) & =-G^{-1}\left(\left(\varphi *_{0} \psi\right)^{\prime}\right) \\
& =-G^{-1}\left(\varphi *_{0} \psi^{\prime}+\psi(0) \varphi\right) \\
& =-\left(G^{-1}(\varphi) G\left(\psi^{\prime}\right)+G(\varphi) G^{-1}\left(\psi^{\prime}\right)+\delta(\psi) G^{-1}(\varphi)\right) \\
& =G^{-1}(\varphi) G^{\prime}(\psi)+G(\varphi) G(\psi)-\delta(\psi) G^{-1}(\varphi), \varphi, \psi \in \mathcal{D},
\end{aligned}
$$


and (ii) is satisfied. With assumed (ii), one obtains (iii) from the computation

$$
\begin{aligned}
G^{\prime}\left(\varphi *_{0} \psi\right) & =-G\left(\left(\varphi *_{0} \psi\right)^{\prime}\right)=-G\left(\varphi^{\prime} *_{0} \psi+\varphi(0) \psi\right) \\
& =-\left(G\left(\varphi^{\prime}\right) G(\psi)+G^{-1}\left(\varphi^{\prime}\right)\left(G^{\prime}-\delta\right)(\psi)+\delta(\varphi) G(\psi)\right) \\
& =\left(G^{\prime}-\delta\right)(\varphi) G(\psi)+G(\varphi)\left(G^{\prime}-\delta\right)(\psi), \varphi, \psi \in \mathcal{D} .
\end{aligned}
$$

It is also clear that $\mathcal{G}$ satisfies $\left(d_{2}\right)$ if $G$ satisfies $\left(D C F_{2}\right)$. Suppose that $\mathcal{G}$ satisfies $\left(d_{2}\right)$. Let us prove that $G$ satisfies $\left(D C F_{2}\right)$. So let us assume that for some fixed $x, y \in E$, we have $G(\varphi) x+G^{-1}(\varphi) y=0, \varphi \in \mathcal{D}_{0}$. Then

$$
\left(G^{\prime}-\delta\right)(\varphi) x+G(\varphi) y=-G\left(\varphi^{\prime}\right) x-\varphi(0) x-G^{-1}\left(\varphi^{\prime}\right) y=0, \varphi \in \mathcal{D}_{0} .
$$

Since $\mathcal{G}$ satisfies $\left(d_{2}\right)$, it follows that $x=y=0$.

Note that the previous proposition is motivated by Proposition 1.3. Properties $\left(D C F_{1}\right)$ and $\left(D C F_{2}\right)$ can be interpreted respectively as $\sin (\alpha+\beta) \equiv \sin \alpha \cos \beta+$ $\cos \alpha \sin \beta$, and the linear independence of $\cos (\cdot)$ and $\sin (\cdot)$. Next, we characterize distribution cosine functions as follows.

Proposition 2.3. Let $G \in \mathcal{D}_{0}^{\prime}(L(E))$. Then $G$ is a (DCF) if and only if $\left(D C F_{2}\right)$ holds and

$$
G^{-1}\left(\varphi * \psi_{+}\right)=G^{-1}(\varphi) G(\psi)+G(\varphi) G^{-1}(\psi), \varphi \in \mathcal{D}_{0}, \quad \psi \in \mathcal{D} .
$$

Proof. Assume $G$ is a (DCF). Then $\mathcal{G}$ is a (DSG) in $E^{2}$ and the use of Proposition 1.8 gives $\mathcal{G}\left(\psi_{+}\right)=\mathcal{G}(\psi), \psi \in \mathcal{D}$. Thus

$$
\begin{aligned}
& \left(\begin{array}{cc}
G\left(\varphi * \psi_{+}\right) & G^{-1}\left(\varphi * \psi_{+}\right) \\
\left(G^{\prime}-\delta\right)\left(\varphi * \psi_{+}\right) & G\left(\varphi * \psi_{+}\right)
\end{array}\right)\left(\begin{array}{l}
x \\
y
\end{array}\right) \\
= & \left(\begin{array}{cc}
G(\varphi) & G^{-1}(\varphi) \\
\left(G^{\prime}-\delta\right)(\varphi) & G(\varphi)
\end{array}\right)\left(\begin{array}{cc}
G(\psi) & G^{-1}(\psi) \\
\left(G^{\prime}-\delta\right)(\psi) & G(\psi)
\end{array}\right)\left(\begin{array}{l}
x \\
y
\end{array}\right), \\
& \varphi \in \mathcal{D}_{0}, \psi \in \mathcal{D}, x, y \in E .
\end{aligned}
$$

Choose $x=0$ to obtain (1).

Let us suppose that $\left(D C F_{2}\right)$ and $(1)$ are valid. Then $\mathcal{G}$ satisfies $\left(d_{2}\right)$. Assumption (1) implies $G^{-1}(\varphi * \psi)=G^{-1}(\varphi) G(\psi)+G(\varphi) G^{-1}(\psi), \varphi, \psi \in \mathcal{D}_{0}$, and consequently,

$$
\begin{aligned}
& G(\varphi * \psi)=G(\varphi) G(\psi)+G^{-1}(\varphi)\left(G^{\prime}-\delta\right)(\psi), \\
& \left(G^{\prime}-\delta\right)(\varphi * \psi)=\left(G^{\prime}-\delta\right)(\varphi) G(\psi)+G(\varphi)\left(G^{\prime}-\delta\right)(\psi), \varphi, \psi \in \mathcal{D}_{0} .
\end{aligned}
$$


So $\left(d_{1}\right)$ holds for $\mathcal{G}$. Then

$$
\begin{aligned}
& G\left(\varphi * \psi_{+}\right)=-G^{-1}\left(\left(\varphi *_{0} \psi_{+}\right)^{\prime}\right. \\
= & -G^{-1}\left(\varphi^{\prime} *_{0} \psi_{+}+\varphi(0) \psi_{+}\right)=-\left[G^{-1}\left(\varphi^{\prime}\right) G(\psi)+G\left(\varphi^{\prime}\right) G^{-1}(\psi)\right] \\
= & G(\varphi) G(\psi)+G^{\prime}(\varphi) G^{-1}(\psi), \varphi \in \mathcal{D}_{0}, \psi \in \mathcal{D} .
\end{aligned}
$$

Since $\left(\varphi *_{0} \psi_{+}\right)^{\prime}=\left(\varphi *_{0}\left(\psi^{\prime}\right)_{+}\right)+\psi(0) \varphi, \varphi \in \mathcal{D}_{0}, \psi \in \mathcal{D}$, we have

$$
\begin{aligned}
& G\left(\varphi *_{0} \psi_{+}\right)=-G^{-1}\left(\left(\varphi *_{0} \psi_{+}\right)^{\prime}\right)=-G^{-1}\left(\varphi *_{0}\left(\psi^{\prime}\right)_{+}+\psi(0) \varphi\right) \\
(3) \quad= & {\left[G^{-1}(\varphi) G\left(\psi^{\prime}\right)+G(\varphi) G^{-1}\left(\psi^{\prime}\right)\right]-\psi(0) G^{-1}(\varphi) } \\
= & G(\varphi) G(\psi)+G^{-1}(\varphi)\left(G^{\prime}-\delta\right)(\psi), \varphi \in \mathcal{D}_{0}, \psi \in \mathcal{D}, \text { and } \\
& \left(G^{\prime}-\delta\right)\left(\varphi * \psi_{+}\right)=G^{\prime}\left(\varphi * \psi_{+}\right)=-G\left(\left(\varphi *_{0} \psi_{+}\right)^{\prime}\right)=-G\left(\varphi^{\prime} *_{0} \psi_{+}\right) \\
(4) \quad & \stackrel{(3)}{=}-\left[G\left(\varphi^{\prime}\right) G(\psi)+G^{-1}\left(\varphi^{\prime}\right)\left(G^{\prime}-\delta\right)(\psi)\right] \\
= & \left(G^{\prime}-\delta\right)(\varphi) G(\psi)+G(\varphi)\left(G^{\prime}-\delta\right)(\psi), \varphi \in \mathcal{D}_{0}, \psi \in \mathcal{D} .
\end{aligned}
$$

Thus, $\left(d_{5}\right)$ holds for $\mathcal{G}$ and $\mathcal{G}$ is a (DSG) in $E^{2}$. The rest of proof is clear by Proposition 2.2.

Proposition 2.4. Let $G \in \mathcal{D}_{0}^{\prime}(L(E))$. Then $G$ is a pre-(DCF) if and only if

$$
G^{-1}(\varphi)\left(G^{\prime}-\delta\right)(\psi)=\left(G^{\prime}-\delta\right)(\varphi) G^{-1}(\psi), \varphi, \psi \in \mathcal{D} .
$$

Proof. We have proved (see Proposition 2.2) that $G$ is a pre-(DCF) in $E$ if and only if $\mathcal{G}$ is a pre-(DSG) in $E^{2}$. The use of [7, Proposition 2] gives that anyone of these conditions is also equivalent to

$$
\mathcal{G}\left(\varphi^{\prime}\right) \mathcal{G}(\psi)-\mathcal{G}(\varphi) \mathcal{G}\left(\psi^{\prime}\right)=\psi(0) \mathcal{G}(\varphi)-\varphi(0) \mathcal{G}(\psi), \varphi, \psi \in \mathcal{D}
$$

As in the proofs of Proposition 2.2 and Proposition 2.3, one can prove: (6) holds if and only if (5) holds.

Infinitesimal generator of a (DCF) can be defined in several different ways; here we follow ideas in [9] and [13] where the generator of a (DSG) [(L-DSG)] is introduced.

Definition 2.5. The generator $A$ of a (DCF) $G$ is given by

$$
\left\{(x, y) \in E^{2}:\left(\forall \varphi \in \mathcal{D}_{0}\right) G^{-1}\left(\varphi^{\prime \prime}\right) x=G^{-1}(\varphi) y\right\} .
$$


Because of $\left(D C F_{2}\right), A$ is a function and it is easy to see that $A$ is a closed linear operator in $E$.

Lemma 2.6. Let $A$ be the generator of a (DCF) $G$. Then $\mathcal{A} \subset \mathcal{B}$, where $\mathcal{A} \equiv\left(\begin{array}{cc}0 & I \\ A & 0\end{array}\right)$ and $\mathcal{B}$ is the generator of $\mathcal{G}$. Moreover, $(x, y) \in A \Leftrightarrow\left(\left(\begin{array}{l}x \\ 0\end{array}\right),\left(\begin{array}{l}0 \\ y\end{array}\right)\right) \in \mathcal{B}$.

Proof. Let $\left(\left(\begin{array}{l}x \\ y\end{array}\right),\left(\begin{array}{l}u \\ v\end{array}\right)\right) \in \mathcal{A}$. Then $x \in D(A), y=u$ and $A x=v$. We have to prove that for all $\varphi \in \mathcal{D}_{0}, \mathcal{G}\left(-\varphi^{\prime}\right)\left(\begin{array}{l}x \\ y\end{array}\right)=\mathcal{G}(\varphi)\left(\begin{array}{l}u \\ v\end{array}\right)$. Let $\varphi \in \mathcal{D}_{0}$ be fixed. The definition of $A$ implies

$$
G\left(-\varphi^{\prime}\right) x=G^{-1}\left(\varphi^{\prime \prime}\right) x=G^{-1}(\varphi) A x=G^{-1}(\varphi) v,
$$

and

$$
G^{\prime}\left(-\varphi^{\prime}\right) x=-G^{-1}\left(\varphi^{\prime \prime \prime}\right) x=-G^{-1}\left(\varphi^{\prime}\right) A x=-G^{-1}\left(\varphi^{\prime}\right) v=G(\varphi) v .
$$

Since $\varphi(0)=\varphi^{\prime}(0)=0$ and $y=u$, we obtain

$$
\begin{aligned}
& G\left(-\varphi^{\prime}\right) x+G^{-1}\left(-\varphi^{\prime}\right) y=G(\varphi) u+G^{-1}(\varphi) v, \text { and } \\
& \left(G^{\prime}-\delta\right)\left(-\varphi^{\prime}\right) x+G\left(-\varphi^{\prime}\right) y=\left(G^{\prime}-\delta\right)(\varphi) u+G(\varphi) v .
\end{aligned}
$$

It implies $-\mathcal{G}\left(\varphi^{\prime}\right)\left(\begin{array}{l}x \\ y\end{array}\right)=\mathcal{G}(\varphi)\left(\begin{array}{l}u \\ v\end{array}\right), \varphi \in \mathcal{D}_{0}$, and $\left(\left(\begin{array}{l}x \\ y\end{array}\right),\left(\begin{array}{l}u \\ v\end{array}\right)\right) \in \mathcal{B}$. Assume that $(x, y) \in A$. Then $\left(\left(\begin{array}{l}x \\ 0\end{array}\right),\left(\begin{array}{l}0 \\ y\end{array}\right)\right) \in \mathcal{A}$, and consequently, $\left(\left(\begin{array}{l}x \\ 0\end{array}\right),\left(\begin{array}{l}0 \\ y\end{array}\right)\right) \in \mathcal{B}$. Suppose now that $\left(\left(\begin{array}{l}x \\ 0\end{array}\right),\left(\begin{array}{l}0 \\ y\end{array}\right)\right) \in \mathcal{B}$. Let us fix $\varphi \in \mathcal{D}_{0}$. Then $\mathcal{G}\left(-\varphi^{\prime}\right)\left(\begin{array}{l}x \\ 0\end{array}\right)=\mathcal{G}(\varphi)\left(\begin{array}{l}0 \\ y\end{array}\right)$, and by the definition of $\mathcal{G}$,

$$
\left(\begin{array}{cc}
G\left(-\varphi^{\prime}\right) & G^{-1}\left(-\varphi^{\prime}\right) \\
G^{\prime}\left(-\varphi^{\prime}\right)-\delta\left(-\varphi^{\prime}\right) & G\left(-\varphi^{\prime}\right)
\end{array}\right)\left(\begin{array}{l}
x \\
0
\end{array}\right)=\left(\begin{array}{cc}
G(\varphi) & G^{-1}(\varphi) \\
G^{\prime}(\varphi)-\delta(\varphi) & G(\varphi)
\end{array}\right)\left(\begin{array}{l}
0 \\
y
\end{array}\right)
$$

It implies $G\left(-\varphi^{\prime}\right) x=G^{-1}(\varphi) y$, i.e., $G^{-1}\left(\varphi^{\prime \prime}\right) x=G^{-1}(\varphi) y$. Hence, $(x, y) \in A$. The proof is completed.

We will prove in Theorem 3.10(ii) that $\mathcal{A}=\mathcal{B}$.

The following proposition will help to get relations between distribution cosine functions and local integrated cosine functions. Property $\left(D C F_{2}\right)$ has an important role again.

Proposition 2.7. Let $G$ be a (DCF) generated by A. Then

(i) $\left(G(\psi) x, G\left(\psi^{\prime \prime}\right) x+\psi^{\prime}(0) x\right) \in A, \psi \in \mathcal{D}, x \in E$. 
(ii) $\left(G^{-1}(\psi) x,-G\left(\psi^{\prime}\right) x-\psi(0) x\right) \in A, \psi \in \mathcal{D}, x \in E$.

(iii) $G(\psi) A \subset A G(\psi), \psi \in \mathcal{D}$.

(iv) $G^{-1}(\psi) A \subset A G^{-1}(\psi), \psi \in \mathcal{D}$.

Proof. Let $x, y \in E$.

(i) Clearly, $(G(\psi) x, y) \in A$ if and only if $G^{\prime}(\varphi) G(\psi) x=G^{-1}(\varphi) y, \varphi \in \mathcal{D}$. If $\varphi \in \mathcal{D}_{0}$ then $\varphi(0)=0$ and by (iii) in the proof of Proposition 2.2, this is equivalent to:

$$
\begin{gathered}
G^{\prime}\left(\varphi *_{0} \psi\right) x-G(\varphi) G^{\prime}(\psi) x+\psi(0) G(\varphi) x=G^{-1}(\varphi) y, \varphi \in \mathcal{D}_{0} \\
\Leftrightarrow \\
-G\left(\varphi *_{0} \psi^{\prime}+\psi(0) \varphi\right) x-G(\varphi) G^{\prime}(\psi) x+\psi(0) G(\varphi) x=G^{-1}(\varphi) y, \varphi \in \mathcal{D}_{0} \\
\Leftrightarrow \\
\Leftrightarrow\left(\varphi *_{0} \psi^{\prime}\right) x-G(\varphi) G^{\prime}(\psi) x=G^{-1}(\varphi) y, \varphi \in \mathcal{D}_{0} .
\end{gathered}
$$

By (ii) in the proof of Proposition 2.2, this is equivalent to

$$
\begin{gathered}
-\left[G(\varphi) G\left(\psi^{\prime}\right) x+G^{-1}(\varphi)\left(G^{\prime}\left(\psi^{\prime}\right) x-\psi^{\prime}(0) x\right)\right]-G(\varphi) G^{\prime}(\psi) x=G^{-1}(\varphi) y, \varphi \in \mathcal{D}_{0} \\
\Leftrightarrow \\
G(\varphi)\left[-G\left(\psi^{\prime}\right) x-G^{\prime}(\psi) x\right]+G^{-1}(\varphi)\left[-G^{\prime}\left(\psi^{\prime}\right) x+\psi^{\prime}(0) x-y\right]=0, \varphi \in \mathcal{D}_{0} \\
\Leftrightarrow \\
y=G\left(\psi^{\prime \prime}\right) x+\psi^{\prime}(0) x .
\end{gathered}
$$

(ii) Let us recall that $G^{-1}(\psi)=-G(I(\psi))$ and that $\frac{d}{d t} I(\psi)(t)=\psi(t)-$ $\alpha(t) \int_{-\infty}^{\infty} \psi(u) d u, t \in \mathbb{R}$. Hence, $\frac{d^{2}}{d t^{2}} I(\psi)(t)=\psi^{\prime}(t)-\alpha^{\prime}(t) \int_{-\infty}^{\infty} \psi(u) d u, t \in$ $\mathbb{R}$. Since $\alpha \in \mathcal{D}_{[-2,-1]}$ and $G \in \mathcal{D}_{0}^{\prime}(L(E))$, we obtain $(I(\psi))^{\prime}(0)=\psi(0)$ and $G\left((I(\psi))^{\prime \prime}\right)=G\left(\psi^{\prime}-\alpha^{\prime} \int_{-\infty}^{\infty} \psi(u) d u\right)=G\left(\psi^{\prime}\right)$. The use of (i) gives $A G^{-1}(\psi) x=-A G(I(\psi)) x=-\left[G\left((I(\psi))^{\prime \prime}\right) x+(I(\psi))^{\prime}(0) x\right]=-G\left(\psi^{\prime}\right) x-\psi(0) x$.

(iii) Let $x \in D(A)$. Then $\left(\left(\begin{array}{c}x \\ 0\end{array}\right),\left(\begin{array}{c}0 \\ A x\end{array}\right)\right) \in \mathcal{A}$ and Lemma 2.6 implies $\left(\left(\begin{array}{c}x \\ 0\end{array}\right),\left(\begin{array}{c}0 \\ A x\end{array}\right)\right) \in$ $\mathcal{B}$, where $\mathcal{B}$ is the generator of $\mathcal{G}$. By [9, Lemma 3.6], we obtain

$$
\mathcal{G}(\psi)\left(\begin{array}{c}
0 \\
A x
\end{array}\right)=-\mathcal{G}\left(\psi^{\prime}\right)\left(\begin{array}{l}
x \\
0
\end{array}\right)-\psi(0)\left(\begin{array}{l}
x \\
0
\end{array}\right)
$$

which gives $G(\psi) A x=G\left(\psi^{\prime \prime}\right) x+\psi^{\prime}(0) x$. Thus, (iii) is a consequence of (i). Let us prove (iv). Let $\psi \in \mathcal{D}$; the use of (iii) implies $G^{-1}(\psi) A=$ $-G(I(\psi)) A \subset-A G(I(\psi))=A G^{-1}(\psi)$ and the proof is completed. 
3. Connections with Integrated Cosine Functions, Convolution Equations and Local $C$-Cosine Functions

Basic properties of distribution cosine functions discussed in the preceding section will be frequently used. We will show that every generator of a (DCF) generates a local integrated cosine function and that the reverse implication is also true.

In the next theorem, we follow the proof of [1, Theorem 7.2] with appropriate changes.

Theorem 3.1. Let $A$ be the generator of a (DCF) $G$. Then there exist $\tau>0$, $n \in \mathbb{N}$ and a local n-times integrated cosine function $\left(C_{n}(t)\right)_{t \in[0, \tau)}$ generated by A.

Proof. By Proposition 2.7(i), we have for every $\varphi \in \mathcal{D}$ and $x \in E, A G(\varphi) x=$ $G\left(\varphi^{\prime \prime}\right) x+\varphi^{\prime}(0) x$. This implies that $G$ is a continuous linear mapping from $\mathcal{D}$ into $L(E,[D(A)])$. By [14, Theorem 2.1.1], there are $\tau>0, n \in \mathbb{N}$ and a strongly continuous function $C_{n}:[-\tau, \tau] \rightarrow L(E,[D(A)])$ such that

$$
G(\varphi) x=(-1)^{n} \int_{-\tau}^{\tau} \varphi^{(n)}(t) C_{n}(t) x d t
$$

for all $x \in E, \varphi \in \mathcal{D}_{(-\tau, \tau)}$. Moreover, $\operatorname{supp} G \subset[0, \infty)$ implies $C_{n}(t)=0$ on $(-\infty, 0)$, and

$$
\begin{aligned}
& (-1)^{n} \int_{0}^{\tau} \varphi^{(n)}(t) A C_{n}(t) x d t=A G(\varphi) x=G\left(\varphi^{\prime \prime}\right) x+\varphi^{\prime}(0) x \\
= & (-1)^{n+2} \int_{0}^{\tau} \varphi^{(n+2)}(t) C_{n}(t) x d t+\varphi^{\prime}(0) x,
\end{aligned}
$$

for all $x \in E, \varphi \in \mathcal{D}_{(-\tau, \tau)}$. Thus, there exist $B_{0}, \ldots, B_{n+1} \in L(E)$ (cf. [5, Lemma 8.1.1]) such that

$$
\int_{0}^{t}(t-s) A C_{n}(s) x d s-C_{n}(t) x=\sum_{j=0}^{n+1} t^{j} B_{j} x, x \in E, t \in[0, \tau) .
$$

Hence,

$$
(-1)^{n+2} \int_{0}^{\tau} \varphi^{(n+2)}(t) \sum_{j=0}^{n+1} t^{j} B_{j} x d t=\varphi^{\prime}(0) x, \varphi \in \mathcal{D}_{(-\tau, \tau)}, x \in E \text {, i.e. }
$$




$$
(-1)^{n+2} \sum_{j=0}^{n+1}(-1)^{j+1} j ! \varphi^{(n+1-j)}(0) B_{j} x=\varphi^{\prime}(0) x, \varphi \in \mathcal{D}_{(-\tau, \tau)}, x \in E .
$$

One can choose a sequence $\left(\varphi_{k}\right)_{k \in \mathbb{N}_{0}}$ in $\mathcal{D}_{(-\tau, \tau)}$ with $\varphi_{k}^{(j)}(0)=\delta_{j k}, j, k \in \mathbb{N}_{0}$, to conclude that $B_{j}=0, j \in\{0,1, \ldots, n+1\} \backslash\{n\}, B_{n}=\frac{(-1)}{n !} I$, and that

$$
A \int_{0}^{t}(t-s) C_{n}(s) x d s=C_{n}(t) x-\frac{t^{n}}{n !} x, x \in E, t \in[0, \tau) .
$$

Since $G(\varphi)$ commutes with $A, \varphi \in \mathcal{D}$, it follows that there exist $F_{0}, \ldots, F_{n-1} \in$ $L(E)$ such that

$$
A C_{n}(t) x-C_{n}(t) A x=\sum_{j=0}^{n-1} t^{j} F_{j} x, x \in D(A), t \in[0, \tau) .
$$

Arguing similarly as in the first part of the proof, one has $F_{j}=0,0 \leq j \leq n-1$, and $\left(C_{n}(t)\right)_{t \in[0, \tau)}$ is a local $n$-times integrated cosine function generated by $A$.

Let us notice that the proof of converse statement is essentially the same as in [1, Theorem 7.2]. This theorem is slightly changed by Wang [17], where he proved its analogous version, but without any denseness assumption; the same result is shown in [9] using the different approach.

Theorem 3.2. Let $A$ be the generator of a (local) n-times integrated cosine function $\left(C_{n}(t)\right)_{t \in[0, \tau)}$. Then $A$ is the generator of a $(\mathrm{DCF})$.

Proof. It is clear that $\mathcal{A}$ is the generator of an $(n+1)$-times integrated semigroup $\left(S_{n+1}(t)\right)_{t \in[0, \tau)}$, where $S_{n+1}$ is given in Proposition 1.3. By [1, Theorem 4.1] and induction, one can prove that for every $k \in \mathbb{N}, \mathcal{A}$ is the generator of a $\left(2^{k}(n+1)\right)$ times integrated semigroup $\left(S_{2^{k}(n+1)}(t)\right)_{t \in\left[0,2^{k} \tau\right)}$, see also the short discussion after Lemma 1.9. Denote

$$
S_{2^{k}(n+1)}(t)=\left(\begin{array}{cc}
S_{2^{k}(n+1)}^{1}(t) & S_{2^{k}(n+1)}^{2}(t) \\
S_{2^{k}(n+1)}^{3}(t) & S_{2^{k}(n+1)}^{4}(t)
\end{array}\right), 0 \leq t<2^{k} \tau .
$$

The proof of the part (ii) $\Rightarrow$ (i) of Proposition 1.3 gives:

$$
S_{2^{k}(n+1)}^{1}(t)=S_{2^{k}(n+1)}^{4}(t), S_{2^{k}(n+1)}^{2}(t)=\int_{0}^{t} S_{2^{k}(n+1)}^{1}(s) d s
$$


and

$$
S_{2^{k}(n+1)}^{3}(t)=\frac{d}{d t} S_{2^{k}(n+1)}^{1}(t)-\frac{t^{2^{k}(n+1)-1}}{\left(2^{k}(n+1)-1\right) !} I, 0 \leq t<2^{k} \tau .
$$

Furthermore, by the proof of Proposition 1.3, we have that the operator $A$ must be the generator of a $\left(2^{k}(n+1)-1\right)$-times integrated cosine function $\left(C_{2^{k}(n+1)-1}(t)\right)_{t \in\left[0,2^{k} \tau\right)}$ which is given by $C_{2^{k}(n+1)-1}(t)=S_{2^{k}(n+1)}^{3}(t)+\frac{t^{2^{k}(n+1)-1}}{\left(2^{k}(n+1)-1\right) !} I, t \in\left[0,2^{k} \tau\right)$. It implies $S_{2^{k}(n+1)}^{1}(t)=\int_{0}^{t} C_{2^{k}(n+1)-1}(s) d s, t \in\left[0,2^{k} \tau\right)$, and $\left(S_{2^{k}(n+1)}^{1}(t)\right)_{t \in\left[0,2^{k} \tau\right)}$ is a $\left(2^{k}(n+1)\right)$-times integrated cosine function on $\left[0,2^{k} \tau\right)$.

For $\varphi \in \mathcal{D}$, choose $k \in \mathbb{N}$ such that $\varphi \in \mathcal{D}_{\left(-\infty, 2^{k} \tau\right)}$. Define

$$
\mathcal{G}(\varphi)\left(\begin{array}{l}
x \\
y
\end{array}\right):=(-1)^{2^{k}(n+1)} \int_{0}^{\infty} \varphi^{\left(2^{k}(n+1)\right)}(t) S_{2^{k}(n+1)}(t)\left(\begin{array}{l}
x \\
y
\end{array}\right) d t, x, y \in E
$$

and

$$
G(\varphi) x:=(-1)^{2^{k}(n+1)} \int_{0}^{\infty} \varphi^{\left(2^{k}(n+1)\right)}(t) S_{2^{k}(n+1)}^{1}(t) x d t, x \in E .
$$

One can easily prove that the given definitions are independent of $k \in \mathbb{N}$. Moreover, $\mathcal{G}$ is a (DSG) in $E^{2}$ generated by $\mathcal{A}$; see the proof of [17, Theorem 3.8]. Let $\varphi \in \mathcal{D}_{\left(-\infty, 2^{k} \tau\right)}$ and $x \in E$. Then

$$
\begin{aligned}
& G^{-1}(\varphi) x=-G(I(\varphi)) x=-\int_{0}^{\infty}(I(\varphi))^{\left(2^{k}(n+1)\right)}(t) S_{2^{k}(n+1)}^{1}(t) x d t \\
= & -\int_{0}^{\infty}\left(\varphi^{\left(2^{k}(n+1)-1\right)}(t)-\alpha^{\left(2^{k}(n+1)-1\right)}(t) \int_{-\infty}^{\infty} \varphi(u) d u\right) S_{2^{k}(n+1)}^{1}(t) x d t \\
= & -\int_{0}^{\infty} \varphi^{\left(2^{k}(n+1)-1\right)}(t) S_{2^{k}(n+1)}^{1}(t) x d t=\int_{0}^{\infty} \varphi^{\left(2^{k}(n+1)\right)}(t) \int_{0}^{t} S_{2^{k}(n+1)}^{1}(s) x d s d t \\
= & \int_{0}^{\infty} \varphi^{\left(2^{k}(n+1)\right)}(t) S_{2^{k}(n+1)}^{2}(t) x d t, \text { and } \\
& \left(G^{\prime}-\delta\right)(\varphi) x=-\int_{0}^{\infty} \varphi^{\left(2^{k}(n+1)+1\right)}(t) S_{2^{k}(n+1)}^{1}(t) x d t-\varphi(0) x \\
= & \int_{0}^{\infty} \varphi^{\left(2^{k}(n+1)\right)}(t) \frac{d}{d t} S_{2^{k}(n+1)}^{1}(t) x d t-\int_{0}^{\infty} \varphi^{\left(2^{k}(n+1)\right)}(t) \frac{t^{2^{k}(n+1)-1}}{\left(2^{k}(n+1)-1\right) !} x d t
\end{aligned}
$$




$$
=\int_{0}^{\infty} \varphi^{\left(2^{k}(n+1)\right)}(t) S_{2^{k}(n+1)}^{3}(t) x d t .
$$

This implies $\mathcal{G}(\varphi)=\left(\begin{array}{cc}G(\varphi) & G^{-1}(\varphi) \\ \left(G^{\prime}-\delta\right)(\varphi) & G(\varphi)\end{array}\right), \varphi \in \mathcal{D}$. From Proposition 2.2, it follows that $G$ is a (DCF). If $G$ is generated by $B$, then Lemma 2.6 gives

$$
(x, y) \in B \Leftrightarrow\left(\left(\begin{array}{l}
x \\
0
\end{array}\right),\left(\begin{array}{l}
0 \\
y
\end{array}\right)\right) \in \mathcal{A} \Leftrightarrow(x, y) \in A .
$$

The arguments given above imply the following assertions.

Corollary 3.3. Let $A$ be the generator of an n-times integrated cosine function $\left(C_{n}(t)\right)_{t \geq 0}$. Put $G(\varphi) x=\int_{0}^{\infty} \varphi^{(n)}(t) C_{n}(t) x d t, \varphi \in \mathcal{D}, x \in E$. Then $G$ is $a(\mathrm{DCF})$ generated by $A$.

Corollary 3.4. Let $G$ be a (DCF) generated by A. Then there exist $\tau>0$, $n \in \mathbb{N}$ and a local $n$-times integrated cosine function $\left(C_{n}(t)\right)_{t \in[0, \tau)}$ generated by A such that

$G(\varphi) x=(-1)^{n} \int_{0}^{\tau} \varphi^{(n)}(t) C_{n}(t) x d t$, for all $\varphi \in \mathcal{D}_{(-\infty, \tau)}, x \in E$.

Let us consider now so called incomplete abstract Cauchy problem $\left(A C P_{2}\right)$ :

$$
\left(A C P_{2}\right):\left\{\begin{array}{l}
u^{\prime \prime}(t)=A u(t), 0 \leq t<\tau \\
u(0)=x, u^{\prime}(0)=y
\end{array}\right.
$$

It is said (see [18]) that a function $t \mapsto v(t)$ belonging to $C([0, \tau): E)$ is an $n$-times integrated mild solution of $\left(A C P_{2}\right)$ at $(x, y) \in E^{2}$ if for all $t \in[0, \tau)$, $\int_{0}^{t}(t-s) v(s) d s \in D(A)$ and

$$
A \int_{0}^{t}(t-s) v(s) d s=v(t)-\frac{t^{n}}{n !} x-\frac{t^{n+1}}{(n+1) !} y, t \in[0, \tau) .
$$

Using the same arguments as in [18], one obtains the following proposition.

Proposition 3.5. Let $A$ be a closed operator. Then the following assertions are equivalent:

(i) $A$ is the generator of an n-times integrated cosine function $\left(C_{n}(t)\right)_{t \in[0, \tau)}$.

(ii) For all $(x, y) \in E^{2}$ there exists a unique $n$-times integrated mild solution of $\left(A C P_{2}\right)$. 
The logarithmic region $\tilde{\Lambda}_{\alpha, \beta}$ and the exponential region $E(\alpha, \beta)$ are defined as follows:

$$
\begin{gathered}
\tilde{\Lambda}_{\alpha, \beta}=\{\lambda \in \mathbb{C}: \operatorname{Re} \lambda \geq \alpha+\beta \ln (1+|\lambda|)\}, \alpha, \beta>0, \\
E(\alpha, \beta)=\left\{\lambda \in \mathbb{C}: \operatorname{Re} \lambda \geq \beta,|\operatorname{Im} \lambda| \leq e^{\alpha \operatorname{Re} \lambda}\right\}, \alpha, \beta>0 .
\end{gathered}
$$

Theorem 3.6. Let $A$ be a closed operator. Then the following statements are equivalent:

(i) $A$ is the generator of a (DCF).

(ii) There exist $\tau>0$ and $n \in \mathbb{N}$ such that $A$ is the generator of an n-times integrated cosine function on $[0, \tau)$.

(iii) For every $\tau>0$ there is $n \in \mathbb{N}$ such that $A$ is the generator of an $n$-times integrated cosine function on $[0, \tau)$.

(iv) The operator $\mathcal{A} \equiv\left(\begin{array}{cc}0 & I \\ A & 0\end{array}\right)$ is the generator of a (DSG) in $E^{2}$.

(v) For every $\tau>0$ there is $n \in \mathbb{N}$ such that for all $(x, y) \in E^{2}$ there exists a unique n-times integrated mild solution of $\left(A C P_{2}\right)$.

(vi) There are constants $\alpha, \beta, M>0$ and $n \in \mathbb{N}_{0}$ such that

$$
\tilde{\Lambda}_{\alpha, \beta}^{2}=\left\{\lambda^{2}: \lambda \in \tilde{\Lambda}_{\alpha, \beta}\right\} \subset \rho(A) \text { and }\|R(\lambda: A)\| \leq M(1+|\lambda|)^{n}, \lambda \in \tilde{\Lambda}_{\alpha, \beta}^{2} .
$$

Proof. Implication (i) $\Rightarrow$ (ii) is Theorem 3.1 and implication (ii) $\Rightarrow$ (i) is Theorem 3.2. Assume that (ii) is true. Then the operator $\mathcal{A}$ is the generator of an $(n+1)$-times integrated semigroup $\left(S_{n+1}(t)\right)_{t \in[0, \tau)}$. Thus, by [9, Theorem 4.11], $\mathcal{A}$ generates a (DSG) in $E^{2}$ and (iv) holds. If (iv) holds, then for all $\tau>0$ there is an $n \in \mathbb{N}_{0}$ such that the operator $\mathcal{A}$ generates an $(n+1)$-times integrated semigroup $\left(S_{n+1}(t)\right)_{t \in[0, \tau)}\left(\left[9\right.\right.$, Theorem 4.11]). Fix $\tau>0$ and choose $n \in \mathbb{N}_{0}$ such that $\mathcal{A}$ generates an $(n+1)$-times integrated semigroup $\left(S_{n+1}(t)\right)_{t \in[0, \tau)}$. By Proposition 1.3, $A$ must be the generator of a local $n$-times integrated cosine function on $[0, \tau)$ and (iii) is proved. Implication (iii) $\Rightarrow$ (ii) is trivial. The equivalence of (iii) and (v) is just Proposition 3.5. So we have proved (i) $\Leftrightarrow$ (ii) $\Leftrightarrow$ (iii) $\Leftrightarrow$ (iv) $\Leftrightarrow$ (v). The equivalence of (iv) and (vi) is an easy application of [9, Corollary 3.12] and Lemma 1.10 , where we only have to note that $\|R(\cdot: \mathcal{A})\|_{L\left(E^{2}\right)}$ is polynomially bounded on $\tilde{\Lambda}_{\alpha, \beta}$ if and only if $\|R(\cdot: A)\|_{L(E)}$ is polynomially bounded on $\tilde{\Lambda}_{\alpha, \beta}^{2}$.

Remark. The difference between the logarithmic region $\tilde{\Lambda}_{\alpha, \beta}$ and the exponential region $E(\alpha, \beta)$ is inessential here; more precisely, one may replace (vi) with: 
(vi)' : there are constants $\alpha, \beta, M>0$ and $n \in \mathbb{N}_{0}$ such that:

$$
E^{2}(\alpha, \beta)=\left\{\lambda^{2}: \lambda \in E(\alpha, \beta)\right\} \subset \rho(A)
$$

and

$$
\|R(\lambda: A)\| \leq M(1+|\lambda|)^{n}, \lambda \in E^{2}(\alpha, \beta) .
$$

Indeed, let $\alpha>0$ and $\beta>0$ be fixed. Arguing as in [1, Lemma 2.6], one can prove that $\tilde{\Lambda}_{\alpha, \beta} \subset E\left(\frac{1}{\beta}, \alpha\right)$ and that for every $\alpha^{\prime}>\alpha$ there exists $\beta^{\prime} \geq \beta$ such that $E(\alpha, \beta) \subset \tilde{\Lambda}_{\beta^{\prime}, \frac{1}{\alpha^{\prime}}}$. Furthermore, the logarithmic region $\tilde{\Lambda}_{\alpha, \beta}$ in the formulation of Theorem 3.6 can be replaced with the set $\Lambda_{\alpha, \beta}:=\{\lambda \in \mathbb{C}: \operatorname{Re} \lambda \geq$ $\alpha+\beta \ln (1+|\operatorname{Im} \lambda|)\}$. It follows from the estimate

$$
\tilde{\Lambda}_{\alpha, \beta} \subset \Lambda_{\alpha, \beta} \subset \tilde{\Lambda}_{\left(1+\frac{\ln (1+\alpha)}{\alpha} \beta\right)^{-1} \alpha,\left(1+\frac{\ln (1+\alpha)}{\alpha} \beta\right)^{-1} \beta},
$$

which is proved on page 199 of [7].

Proposition 3.7. Let $A$ be the generator of a (DCF). Then there exists $n \in \mathbb{N}_{0}$ such that A generates a global exponentially bounded n-times integrated semigroup.

Proof. By Theorem 3.6 and the previous remark, we have that there exist $k \in \mathbb{N}$ and constants $M>0, \alpha>0$ and $\beta>0$ such that $E^{2}(\alpha, \beta) \subset \rho(A)$ and that $\|R(\lambda: A)\| \leq M|\lambda|^{k}, \lambda \in E^{2}(\alpha, \beta)$. Suppose $z \in \partial(E(\alpha, \beta))$. If $\operatorname{Re} z=\beta$, then $\operatorname{Re} z^{2} \leq \beta^{2}$. If $z=x \pm i e^{\alpha x}$ for some $x \in[\beta, \infty)$, then $\operatorname{Re} z^{2}=$ $x^{2}-e^{2 \alpha x} \leq M_{1}$, where $M_{1}$ is a suitable constant independent of $x \in[\beta, \infty)$. Put $\omega:=\max \left(\beta^{2}, M_{1}\right)$. Then $\{z \in \mathbb{C}: \operatorname{Re} z>\omega\} \subset E^{2}(\alpha, \beta)$ and $\|R(\lambda: A)\| \leq$ $M|\lambda|^{k}, \operatorname{Re} \lambda>\omega$. By [19, Theorem 1.12], $A$ is the generator of an exponentially bounded $(k+2)$-times integrated semigroup.

Proposition 3.8. Let $A$ be a closed operator such that $A$ and $-A$ generate distribution semigroups. Then $A^{2}$ generates a (DCF).

Proof. Since $A$ generates a (DSG), an application of [9, Corollary 3.12] gives that there exist positive constants $\alpha_{1}$ and $\beta_{1}$ such that $\tilde{\Lambda}_{\alpha_{1}, \beta_{1}} \subset \rho(A)$ and that the resolvent of $A$ is polynomially bounded on $\tilde{\Lambda}_{\alpha_{1}}, \beta_{1}$. Similarly, there exist positive constants $\alpha_{2}$ and $\beta_{2}$ such that $\tilde{\Lambda}_{\alpha_{2}, \beta_{2}} \subset \rho(-A)$ and that the resolvent of $-A$ is polynomially bounded on $\tilde{\Lambda}_{\alpha_{2}, \beta_{2}}$. Put $\alpha:=\max \left(\alpha_{1}, \alpha_{2}\right)$ and $\beta:=\max \left(\beta_{1}, \beta_{2}\right)$. By the definition of the logarithmic region $\tilde{\Lambda}_{\alpha, \beta}$, we obtain

$$
\tilde{\Lambda}_{\alpha_{1}, \beta_{1}} \cap \tilde{\Lambda}_{\alpha_{2}, \beta_{2}} \supset \tilde{\Lambda}_{\alpha, \beta} .
$$

This implies that $\|R(\cdot: A)\|_{L(E)}$ and $\|R(\cdot:-A)\|_{L(E)}$ are polynomially bounded on $\tilde{\Lambda}_{\alpha, \beta}$. We conclude that $\tilde{\Lambda}_{\alpha, \beta}^{2} \subset \rho\left(A^{2}\right)$ and that $2 \lambda R\left(\lambda^{2}: A^{2}\right)=R(\lambda$ : 
$A)+R(\lambda:-A), \lambda \in \tilde{\Lambda}_{\alpha, \beta}$. Clearly, $\left\|R\left(\cdot: A^{2}\right)\right\|_{L(E)}$ is polynomially bounded on $\tilde{\Lambda}_{\alpha, \beta}^{2}$. Theorem 3.6 ends the proof.

Next we want to point out some relations of distribution cosine functions with convolution equations. To do this we use Proposition 2.2 to reduce our investigations to the theory of distribution semigroups. For the convenience of a reader, we will repeat Proposition 1.1 of [9].

Proposition 3.9. ([9, Proposition 1.1]). Suppose $X, Y$ and $Z$ are Banach spaces and $b: X \times Y \rightarrow Z$ is bilinear and continuous. Then there is a unique bilinear, separately continuous mapping $*_{b}: \mathcal{D}_{0}^{\prime}(X) \times \mathcal{D}_{0}^{\prime}(Y) \rightarrow \mathcal{D}_{0}^{\prime}(Z)$ such that

$$
(S \otimes x) *_{b}(T \otimes y)=S * T \otimes b(x, y),
$$

for all $S, T \in \mathcal{D}_{0}^{\prime}$ and $x \in X, y \in Y$. Moreover, this mapping is continuous.

\section{Theorem 3.10.}

(i) Let $A$ be a closed operator and $G \in \mathcal{D}_{0}^{\prime}(L(E))$. Then $G$ is (DCF) generated by $A$ if and only if $G \in \mathcal{D}_{0}^{\prime}(L(E,[D(A)]))$,

$$
G * P=\delta^{\prime} \otimes I d_{[D(A)]} \text { and } P * G=\delta^{\prime} \otimes I d_{E},
$$

where $P:=\delta^{\prime \prime} \otimes I-\delta \otimes A \in \mathcal{D}_{0}^{\prime}(L([D(A)], E)), I \in L([D(A)], E)$ denotes the inclusion $D(A) \rightarrow E,\left(\delta^{(k)} \otimes I d_{[D(A)]}\right)(\varphi) x=(-1)^{k} \varphi^{(k)}(0) x,\left(\delta^{(k)} \otimes\right.$ $I)(\varphi) x=(-1)^{k} \varphi^{(k)}(0) x,(\delta \otimes A)(\varphi) x=\varphi(0) A x, \varphi \in \mathcal{D}, x \in D(A), k \in$ $\mathbb{N}_{0}$ and $\left(\delta^{\prime} \otimes I d_{E}\right)(\varphi) x=-\varphi^{\prime}(0) x, \varphi \in \mathcal{D}, x \in E$.

(ii) Let $G \in \mathcal{D}_{0}^{\prime}(L(E))$. Then $G$ is a (DCF) in $E$ generated by $A$ if and only if $\mathcal{G} \equiv\left(\begin{array}{cc}G & G^{-1} \\ G^{\prime}-\delta & G\end{array}\right)$ is a (DSG) in $E^{2}$ generated by $\mathcal{A} \equiv\left(\begin{array}{cc}0 & I \\ A & 0\end{array}\right)$.

Proof. (i) Let $X=L(E,[D(A)]), Y=L([D(A)], E), Z=L([D(A)])$ and $b: X \times Y \rightarrow Z, b(B, C):=B C, B \in X, C \in Y$. The definition of $G * P$ is given by Proposition 3.9; convolution $P * G$ can be understood similarly. Let $x \in D(A), k \in \mathbb{N}_{0}, \varphi \in \mathcal{D}$. Then it can be proved that

$\left(G *\left(\delta^{(k)} \otimes I\right)\right)(\varphi) x=(-1)^{k} G\left(\varphi^{(k)}\right) x,\left(G *\left(\delta^{(k)} \otimes A\right)\right)(\varphi) x=(-1)^{k} G\left(\varphi^{(k)}\right) A x$.

Similarly,

$$
\begin{aligned}
\left(\left(\delta^{(k)} \otimes I\right) * G\right)(\varphi) x & =(-1)^{k} G\left(\varphi^{(k)}\right) x, \quad\left(\left(\delta^{(k)} \otimes A\right) * G\right)(\varphi) x \\
& =(-1)^{k} A G\left(\varphi^{(k)}\right) x, \varphi \in \mathcal{D}, x \in E, \quad k \in \mathbb{N}_{0}
\end{aligned}
$$


Suppose that $G$ is a (DSG) generated by $A$ and $x \in E$. Then Proposition 2.7(i) gives $A G(\varphi) x=G\left(\varphi^{\prime \prime}\right) x+\varphi^{\prime}(0) x$. Consequently, $G \in \mathcal{D}_{0}^{\prime}(L(E,[D(A)]))$,

$$
(P * G)(\varphi) x=G\left(\varphi^{\prime \prime}\right) x-A G(\varphi) x=-\varphi^{\prime}(0) x \text { and } P * G=\delta^{\prime} \otimes I d_{E} .
$$

Analogously, $G * P=\delta^{\prime} \otimes I d_{[D(A)]}$. So let $G \in \mathcal{D}_{0}^{\prime}(L(E,[D(A)]))$ satisfy $G * P=$ $\delta^{\prime} \otimes I d_{[D(A)]}$ and $P * G=\delta^{\prime} \otimes I d_{E}$.

Denote $\mathcal{G}=\left(\begin{array}{cc}G & G^{-1} \\ G^{\prime}-\delta & G\end{array}\right)$. Since $\operatorname{supp} G \subset[0, \infty)$, it follows that $\operatorname{supp} G^{-1} \subset[0, \infty)$. Then $\operatorname{supp} \mathcal{G} \subset[0, \infty)$. If $x \in E$, then the assumptions $G * P=\delta^{\prime} \otimes I d_{[D(A)]}$ and $P * G=\delta^{\prime} \otimes I d_{E}$ imply (i) of Proposition 2.7 and $G(\varphi) A x=G\left(\psi^{\prime \prime}\right) x+\psi^{\prime}(0) x, \varphi \in \mathcal{D}, x \in D(A)$. By the proof of Proposition 2.7, one obtains that

$$
\begin{aligned}
A G^{-1}(\varphi) x & =-G\left(\varphi^{\prime}\right) x-\varphi(0) x, \varphi \in \mathcal{D}, x \in E, \text { and } G^{-1}(\varphi) A x \\
& =-G\left(\varphi^{\prime}\right) x-\varphi(0) x, \varphi \in \mathcal{D}, x \in E .
\end{aligned}
$$

It follows that $\mathcal{G} \in \mathcal{D}_{0}^{\prime}\left(L\left(E^{2},[D(\mathcal{A})]\right)\right)$. Let $x \in D(A)$. Then we obtain

$$
\begin{gathered}
-\mathcal{G}\left(\varphi^{\prime}\right)\left(\begin{array}{l}
x \\
y
\end{array}\right)-\mathcal{G}(\varphi) \mathcal{A}\left(\begin{array}{l}
x \\
y
\end{array}\right)=-\mathcal{G}\left(\varphi^{\prime}\right)\left(\begin{array}{l}
x \\
y
\end{array}\right)-\mathcal{G}(\varphi)\left(\begin{array}{c}
y \\
A x
\end{array}\right) \\
=\left(\begin{array}{c}
-G\left(\varphi^{\prime}\right) x-G^{-1}\left(\varphi^{\prime}\right) y-G(\varphi) y-G^{-1}(\varphi) A x \\
-G^{\prime}\left(\varphi^{\prime}\right) x+\varphi^{\prime}(0) x-G\left(\varphi^{\prime}\right) y-G^{\prime}(\varphi) y+\varphi(0) y-G(\varphi) A x
\end{array}\right)=\varphi(0)\left(\begin{array}{l}
x \\
y
\end{array}\right), \varphi \in \mathcal{D} .
\end{gathered}
$$

Similarly, if $x, y \in E$, then $-\mathcal{G}\left(\varphi^{\prime}\right)\left(\begin{array}{l}x \\ y\end{array}\right)-\mathcal{A G}(\varphi)\left(\begin{array}{l}x \\ y\end{array}\right)=\varphi(0)\left(\begin{array}{l}x \\ y\end{array}\right), \varphi \in \mathcal{D}$. This implies

$$
\mathcal{G} * P_{1}=\delta \otimes I d_{[D(\mathcal{A})]} \text { and } P_{1} * \mathcal{G}=\delta \otimes I d_{E^{2}},
$$

where $P_{1}:=\delta^{\prime} \otimes I d-\delta \otimes \mathcal{A} \in \mathcal{D}_{0}^{\prime}\left(L\left([D(\mathcal{A})], E^{2}\right)\right)$ and $I d \in L\left([D(\mathcal{A})], E^{2}\right)$ denotes the inclusion $D(\mathcal{A}) \rightarrow E^{2}$. This combined with the proof of $[9$, Theorem 3.10] gives that $\mathcal{G}$ is a (DSG) in $E^{2}$ generated by $\mathcal{A}$. Thus, $G$ is a (DCF) in $E$. If $B$ is the generator of $G$, then

$$
(x, y) \in B \Leftrightarrow\left(\left(\begin{array}{l}
x \\
0
\end{array}\right),\left(\begin{array}{l}
0 \\
y
\end{array}\right)\right) \in \mathcal{A} \Leftrightarrow(x, y) \in A .
$$

(ii) Suppose that $G$ is a (DCF) generated by $A$. Then, $\mathcal{G}$ is a (DSG) in $E^{2}$. Let $P$ be as in (i). We have

$$
G * P=\delta^{\prime} \otimes I d_{[D(A)]} \text { and } P * G=\delta^{\prime} \otimes I d_{E} .
$$

Then the proof of (i) implies that the generator of $\mathcal{G}$ is $\mathcal{A}$. Conversely, if $\mathcal{G}$ is a (DSG) generated by $\mathcal{A}$, then $G$ is a (DCF). It can be easily seen that the generator of $G$ is $A$. 
Let us recall that $G$ is a (DSG) generated by $A$ if and only if $G$ is a distribution fundamental solution for $A$ (cf. [9, p. 844 and 845]). Since there is at most one distribution fundamental solution for a closed linear operator $A$, it follows that every (DSG) is uniquely determined by its generator. As we mentioned, Kisyński introduced in [7] the generator of a pre-(DSG) $G$; it is a closed linear operator from $E$ into $E / \mathcal{N}(G)$. He proved that every pre-(DSG) is uniquely determined by its generator, see [7, Corollary 2].

Suppose now that $G_{1}$ and $G_{2}$ are distribution cosine functions generated by a closed linear operator $A$. By Theorem 3.10(ii), $\mathcal{G}_{1} \equiv\left(\begin{array}{cc}G_{1} & G_{1}^{-1} \\ G_{1}^{\prime}-\delta & G_{1}\end{array}\right)$ and $\mathcal{G}_{2} \equiv$ $\left(\begin{array}{cc}G_{2} & G_{2}^{-1} \\ G_{2}^{\prime}-\delta & G_{2}\end{array}\right)$ are distribution semigroups generated by $\mathcal{A}$. Consequently, for every $x \in E$ and $\varphi \in \mathcal{D}, \mathcal{G}_{1}(\varphi)\left(\begin{array}{l}x \\ 0\end{array}\right)=\mathcal{G}_{2}(\varphi)\left(\begin{array}{l}x \\ 0\end{array}\right)$. It implies $G_{1}(\varphi) x=G_{2}(\varphi) x$. Hence, we have proved the following assertion:

Corollary 3.11. Every distribution cosine function is uniquely determined by its generator.

Let us consider now the relations between distribution cosine functions and local $C$-cosine functions; we refer to [16] for the introduction to the theory of local $C$-cosine functions.

Proposition 3.12. Let $A$ be a closed operator. Then the following statements are equivalent:

(i) A generates a (DCF),

(ii) $\rho(A) \neq \emptyset$ and there exist $n \in \mathbb{N}$ and $\tau \in(0, \infty]$ such that $A$ is the generator of an $R(\lambda: A)^{n}$-cosine function on $[0, \tau)$, for all $\lambda \in \rho(A)$,

(iii) $\rho(A) \neq \emptyset$ and there exist $\lambda \in \rho(A), n \in \mathbb{N}$ and $\tau \in(0, \infty]$ such that $A$ is the generator of an $R(\lambda: A)^{n}$-cosine function on $[0, \tau)$.

Proof. (i) $\Rightarrow$ (ii) By Theorem 3.6, $\rho(A) \neq \emptyset$ and there exists $\tau \in(0, \infty]$ and $k \in \mathbb{N}$ such that $A$ is the generator of a local $k$-times integrated cosine function on $[0, \tau)$. Without any loss of generality, we can assume that $k=2 n$, for some $n \in \mathbb{N}$. Denote by $\left(C_{2 n}(t)\right)_{t \in[0, \tau)} 2 n$-times integrated cosine function generated by $A$; then the operator $\mathcal{A}$ generates a $(2 n+1)$-times integrated semigroup $\left(S_{2 n+1}(t)\right)_{t \in[0, \tau)}$. Moreover, since $\rho(A) \neq \emptyset$, we have $\rho(\mathcal{A}) \neq \emptyset$ and the use of [1, Proposition 3.3] gives that for all $\left(\begin{array}{ll}x & y\end{array}\right)^{T} \in D\left(\mathcal{A}^{2 n+2}\right)=D\left(A^{n+1}\right) \times D\left(A^{n+1}\right)$ there exists a unique function $U \in C^{1}\left([0, \tau): E^{2}\right) \cap C([0, \tau): D(\mathcal{A}))$ satisfying $U^{\prime}(t)=\mathcal{A} U(t)$, $0 \leq t<\tau$, and $U(0)=\left(\begin{array}{ll}x & y\end{array}\right)^{T}$. Denote $U(t)=\left(\begin{array}{ll}u(t) & v(t)\end{array}\right)^{T}, 0 \leq t<\tau$. We obtain that $u(t) \in D(A), t \in[0, \tau)$ and that $\left(\begin{array}{c}u^{\prime}(t) \\ v^{\prime}(t)\end{array}\right)=\left(\begin{array}{c}v(t) \\ A u(t)\end{array}\right), t \in[0, \tau)$. Hence, the function $t \mapsto u(t)$ belongs to $C^{2}([0, \tau): E) \cap C([0, \tau): D(A))$ and 
it is a solution of the problem $\left(A C P_{2}\right)$ at $\left(\begin{array}{ll}x & y\end{array}\right)^{T} \in D\left(A^{n+1}\right) \times D\left(A^{n+1}\right)$. Suppose that the function $u_{1}$ belonging $C^{2}([0, \tau): E) \cap C([0, \tau): D(A))$ is an another solution of the problem $\left(A C P_{2}\right)$ at $\left(\begin{array}{ll}x & y\end{array}\right)^{T} \in D\left(A^{n+1}\right) \times D\left(A^{n+1}\right)$. Define $U_{1}(t)=\left(\begin{array}{l}u_{1}(t) \\ u_{1}^{\prime}(t)\end{array}\right), t \in[0, \tau)$. Then it can be easily seen that $U_{1} \in C^{1}([0, \tau)$ : $\left.E^{2}\right) \cap C([0, \tau): D(\mathcal{A}))$ and that $U_{1}^{\prime}(t)=\mathcal{A} U_{1}(t), 0 \leq t<\tau$, and $U_{1}(0)=$ $\left(\begin{array}{ll}x & y\end{array}\right)^{T}$. Therefore, $U=U_{1}$, and consequently, $u=u_{1}$. We have proved that there exists a unique function $u \in C^{2}([0, \tau): E) \cap C([0, \tau): D(A))$ which satisfies $\left(A C P_{2}\right)$ for all initial values $(x, y) \in D\left(A^{n+1}\right) \times D\left(A^{n+1}\right)$. Thus, an application of [16, Corollary 8.8], with $C=R(\lambda: A)^{n}$, gives that $A$ generates a (local) $R(\lambda: A)^{n}$ -cosine function on $[0, \tau)$. Hence, (ii) is proved. Implication (ii) $\Rightarrow$ (iii) is trivial. Suppose now that (iii) is valid. Denote by $\left(\bar{C}_{0}(t)\right)_{t \in[0, \tau)}$ a local $R(\lambda: A)^{n}$-cosine function generated by $A$. Let $x, y \in D\left(A^{n+1}\right)$ be fixed and let $u \in C^{2}([0, \tau)$ : $E) \cap C([0, \tau): D(A))$ be a unique (strong) solution of the problem $\left(A C P_{2}\right)$ which is given by [16, Corollary 8.8]. Define $U(t):=\left(\begin{array}{ll}u(t) & u^{\prime}(t)\end{array}\right)^{T}, 0 \leq t<\tau$. Then $U^{\prime}(t)=\left(\begin{array}{cc}u^{\prime}(t) & u^{\prime \prime}(t)\end{array}\right)^{T}=\mathcal{A}\left(u(t) \quad u^{\prime}(t)\right)^{T}=\mathcal{A} U(t), 0 \leq t<\tau$. Clearly, $U(0)=\left(\begin{array}{ll}x & y\end{array}\right)^{T}$ and $U \in C^{1}\left([0, \tau), E^{2}\right)$. Let $0 \leq t, s<\tau$. Since

$$
\begin{gathered}
\|U(t)-U(s)\|_{D(\mathcal{A})}=\left\|\left(\begin{array}{c}
u(t)-u(s) \\
u^{\prime}(t)-u^{\prime}(s)
\end{array}\right)\right\|_{E^{2}}+\left\|\mathcal{A}\left(\begin{array}{c}
u(t)-u(s) \\
u^{\prime}(t)-u^{\prime}(s)
\end{array}\right)\right\|_{E^{2}} \\
=\|u(t)-u(s)\|+2\left\|u^{\prime}(t)-u^{\prime}(s)\right\|+\|A(u(t)-u(s))\|,
\end{gathered}
$$

and $u \in C^{2}([0, \tau): E) \cap C([0, \tau): D(A))$, it follows that $U \in C([0, \tau), D(\mathcal{A}))$. Hence, we have proved that the function $U$ is a solution of the following problem

$$
\left\{\begin{array}{l}
\left.U \in C([0, \tau), D(\mathcal{A})) \cap C^{1}\left([0, \tau), E^{2}\right)\right) \\
U^{\prime}(t)=\mathcal{A} U(t), t \in[0, \tau) \\
U(0)=\left(\begin{array}{ll}
x & y
\end{array}\right)^{T}
\end{array}\right.
$$

Suppose that a function $U_{1}=\left(\begin{array}{l}u_{1} \\ v_{1}\end{array}\right)$ is also a solution of the previous problem. Then it is straightforward to see that $v_{1}=u_{1}^{\prime}$ and that the function $u_{1}$ is also a strong solution of the problem $\left(A C P_{2}\right)$. Consequently, $u=u_{1}$ and $U=U_{1}$. Since $\rho(\mathcal{A}) \neq \emptyset, \mathcal{A}$ generates a local $(2 n+1)$-times integrated semigroup $\left(S_{2 n+1}(t)\right)_{t \in[0, \tau)}$ in $E^{2}$ by virtue of [1, Proposition 3.3]. Proposition 1.3 implies that $A$ generates a local $2 n$-times integrated cosine function $\left(C_{2 n}(t)\right)_{t \in[0, \tau)}$ and an application of Theorem 3.6 gives that (i) holds. The proof is completed.

\section{Exponential Distribution Cosine Functions}

The main aim of this section is to give the fundamental properties of an exponential (DCF). We use the following notation. If $U \in \mathcal{D}^{\prime}(E)$, then we also 
write $\langle U, \varphi\rangle$ for $U(\varphi)$, and $\left\langle e^{a t} U, \varphi\right\rangle$ for $U\left(e^{a \cdot} \varphi\right), a \in \mathbb{R}$. Now we introduce the following definitions.

Definition 4.1. A distribution semigroup $G$ is said to be an exponential distribution semigroup, (EDSG) in short, if there exists $\varepsilon \in \mathbb{R}$ such that $e^{-\varepsilon t} G \in \mathcal{S}^{\prime}(L(E)$ ).

Definition 4.2. A distribution cosine function $G$ is said to be an exponential distribution cosine function, (EDCF) in short, if $\mathcal{G} \equiv\left(\begin{array}{cc}G & G^{-1} \\ G^{\prime}-\delta & G\end{array}\right)$ is an (EDSG) in $E^{2}$.

The following result might be surprising:

Proposition 4.3. Let $G$ be a (DCF). Then $G$ is an (EDCF) if and only if there exists $\varepsilon \in \mathbb{R}$ such that $e^{-\varepsilon t} G^{-1} \in \mathcal{S}_{0}^{\prime}(L(E))$.

Proof. Recall, $\mathcal{S}$ is topologized by the seminorms $\|\psi\|_{\alpha, \beta}:=\sup _{x \in \mathbb{R}} \mid x^{\alpha} \psi^{(\beta)}$ $(x) \mid, \alpha, \beta \in \mathbb{N}_{0}, \psi \in \mathcal{S}$. Assume that $G$ is an (EDCF). By Definition 4.1 and Definition 4.2, we obtain that there exists $\varepsilon \in \mathbb{R}$ such that $e^{-\varepsilon t}\left(\begin{array}{cc}G & G^{-1} \\ G^{\prime}-\delta & G\end{array}\right) \in$ $\mathcal{S}^{\prime}\left(L\left(E^{2}\right)\right)$, i.e., there exists $M>0$ and $\alpha, \beta \in \mathbb{N}_{0}$ such that for every $\varphi \in \mathcal{D}$,

$$
\left\|\left\langle e^{-\varepsilon t}\left(\begin{array}{cc}
G & G^{-1} \\
G^{\prime}-\delta & G
\end{array}\right), \varphi\right\rangle\right\|_{L\left(E^{2}\right)} \leq M\|\varphi\|_{\alpha, \beta} .
$$

Therefore, for all $\varphi \in \mathcal{D}$ and $x, y \in E$, the following holds

$$
\begin{aligned}
& \left\|\left\langle e^{-\varepsilon t} G, \varphi\right\rangle x+\left\langle e^{-\varepsilon t} G^{-1}, \varphi\right\rangle y\right\|+\left\|\left\langle e^{-\varepsilon t}\left(G^{\prime}-\delta\right), \varphi\right\rangle x+\left\langle e^{-\varepsilon t} G, \varphi\right\rangle y\right\| \\
& \leq M\|\varphi\|_{\alpha, \beta}(\|x\|+\|y\|) .
\end{aligned}
$$

Choose $x=0$ to obtain $e^{-\varepsilon t} G^{-1} \in \mathcal{S}_{0}^{\prime}(L(E))$. Suppose now $e^{-\varepsilon t} G^{-1} \in \mathcal{S}_{0}^{\prime}(L(E))$. Then there exist $M>0$ and $\alpha, \beta \in \mathbb{N}_{0}$ so that $\left\|G^{-1}\left(e^{-\varepsilon t} \varphi\right)\right\| \leq M\|\varphi\|_{\alpha, \beta}, \varphi \in$ $\mathcal{D}$. Then

$$
\begin{aligned}
& \left\|\left(e^{-\varepsilon t} G\right)(\varphi)\right\|=\left\|G\left(e^{-\varepsilon t} \varphi\right)\right\|=\left\|G^{-1}\left(-\varepsilon e^{-\varepsilon t} \varphi+e^{-\varepsilon t} \varphi^{\prime}\right)\right\| \\
\leq & M|\varepsilon|\|\varphi\|_{\alpha, \beta}+M\left\|\varphi^{\prime}\right\|_{\alpha, \beta} \\
\leq & M|\varepsilon|\|\varphi\|_{\alpha, \beta}+M\|\varphi\|_{\alpha, \beta+1}, \varphi \in \mathcal{D} .
\end{aligned}
$$

It implies $e^{-\varepsilon t} G \in \mathcal{S}_{0}^{\prime}(L(E))$; similarly, $e^{-\varepsilon t}\left(G^{\prime}-\delta\right) \in \mathcal{S}_{0}^{\prime}(L(E))$ and we obtain

$$
e^{-\varepsilon t}\left(\begin{array}{cc}
G & G^{-1} \\
G^{\prime}-\delta & G
\end{array}\right) \in \mathcal{S}_{0}^{\prime}\left(L\left(E^{2}\right)\right)
$$


Next, let us notice the following fact: if $G$ is a (DSG) generated by $A$ and $\varepsilon \in \mathbb{R}$, then $e^{-\varepsilon t} G$ is a (DSG) generated by $A-\varepsilon I$. The straightforward proof is omitted.

Let $E$ be a Banach space. We need a structural theorem for the space $\mathcal{S}^{\prime}(E)$, see [14, Theorem 2.1.2]: Let $U \in \mathcal{S}^{\prime}(E)$. Then there exist $n \in \mathbb{N}, r>0$ and $a$ continuous function $f: \mathbb{R} \rightarrow E$ such that

$$
U(\varphi)=(-1)^{n} \int_{\mathbb{R}} \varphi^{(n)}(t) f(t) d t
$$

for all $\varphi \in \mathcal{S}$, and $|f(t)|={ }_{|t| \rightarrow \infty} O\left(|t|^{r}\right)$. If $U=0$ on $(-\infty, a)$, then $f(t)=0$ for $t<a$.

Theorem 4.4. Let $A$ be a closed operator. Then the following assertions are equivalent:

(i) $A$ is the generator of an (EDCF) in $E$.

(ii) The operator $\mathcal{A}$ is the generator of an (EDSG) in $E^{2}$.

(iii) $A$ is the generator of a global exponentially bounded n-times integrated cosine function for some $n \in \mathbb{N}$.

(iv) There are constants $\omega, M>0$ and $k \in \mathbb{N}$ such that

$$
\Pi_{\omega}:=\left\{\lambda^{2}: \lambda \in \mathbb{C}, \operatorname{Re} \lambda>\omega\right\} \text { and }\|R(\lambda: A)\| \leq M|\lambda|^{k}, \lambda \in \Pi_{\omega} .
$$

Proof. Implication (i) $\Rightarrow$ (ii) follows immediately from Theorem 3.10(ii). We show (ii) $\Rightarrow$ (iii). Let us suppose that $\mathcal{G}$ is a (DSG) in $E^{2}$ generated by $\mathcal{A}$ and $e^{-\varepsilon t} \mathcal{G} \in \mathcal{S}_{0}^{\prime}\left(L\left(E^{2}\right)\right)$. It implies that $e^{-\varepsilon t} \mathcal{G}$ is a (DSG) in $E^{2}$ generated by $\mathcal{A}-$ $\left(\begin{array}{cc}\varepsilon I & 0 \\ 0 & \varepsilon I\end{array}\right)$. By [9, Lemma 3.6], for every $\varphi \in \mathcal{D}$ and $x, y \in E$ :

$$
\mathcal{A}\left\langle e^{-\varepsilon t} \mathcal{G}, \varphi\right\rangle\left(\begin{array}{l}
x \\
y
\end{array}\right)=\left\langle e^{-\varepsilon t} \mathcal{G},-\varphi^{\prime}\right\rangle\left(\begin{array}{l}
x \\
y
\end{array}\right)+\varepsilon\left\langle e^{-\varepsilon t} \mathcal{G}, \varphi\right\rangle\left(\begin{array}{l}
x \\
y
\end{array}\right)-\varphi(0)\left(\begin{array}{l}
x \\
y
\end{array}\right),
$$

which gives that $e^{-\varepsilon t} \mathcal{G} \in \mathcal{S}^{\prime}\left(L\left(E^{2},[D(\mathcal{A})]\right)\right)$. The use of [14, Theorem 2.1.2] gives that there exist $n \in \mathbb{N}, r>0$ and a continuous function $\bar{S}_{n+1}: \mathbb{R} \rightarrow L\left(E^{2},[D(\mathcal{A})]\right)$ supported by $[0, \infty)$ such that

$$
\left\langle e^{-\varepsilon t} \mathcal{G}, \varphi\right\rangle\left(\begin{array}{l}
x \\
y
\end{array}\right)=(-1)^{n+1} \int_{0}^{\infty} \varphi^{(n+1)}(t) \bar{S}_{n+1}(t)\left(\begin{array}{l}
x \\
y
\end{array}\right) d t
$$

for all $\varphi \in \mathcal{D}, x, y \in E$, and $\left|\bar{S}_{n+1}(t)\right| \leq M t^{r}, t \geq 0$. Arguing similarly as in [1, Theorem 7.2], one has that $\left(\bar{S}_{n+1}(t)\right)_{t \geq 0}$ is an $(n+1)$-times integrated semigroup generated by $\mathcal{A}-\left(\begin{array}{cc}\varepsilon I & 0 \\ 0 & \varepsilon I\end{array}\right)$. The standard perturbation argument (as 
in [1, Lemma 3.2]) shows that $\mathcal{A}$ is the generator of a global exponentially bounded $(n+1)$-times integrated semigroup $\left(S_{n+1}(t)\right)_{t \geq 0}$. Note here that Proposition 1.3 gives that the operator $A$ generates a global $n$-times integrated cosine function $\left(C_{n}(t)\right)_{t \geq 0}$. By an observation given after the proof of Proposition 1.3, we have that $\left(C_{n}(t)\right)_{t \geq 0}$ is exponentially bounded. This proves that (iii) holds. We continue with the proof of (ii) $\Rightarrow$ (i). We will apply the arguments given in the later part of the proof of Theorem 3.2. Namely, it is easy to see that

$$
\mathcal{G}(\varphi)=(-1)^{n+1} \int_{0}^{\infty} \varphi^{(n+1)}(t) S_{n+1}(t) d t, \varphi \in \mathcal{D} .
$$

Define $G$ by

$$
G(\varphi) x:=(-1)^{(n+1)} \int_{0}^{\infty} \varphi^{(n+1)}(t) S_{(n+1)}^{1}(t) x d t, x \in E, \varphi \in \mathcal{D},
$$

with the same terminology as in the proof of Theorem 3.2. Applying the same arguments as in the proof of Theorem 3.2, one obtains $\mathcal{G}=\left(\begin{array}{cc}G & G^{-1} \\ G^{\prime}-\delta & G\end{array}\right)$. Therefore, $G$ is an (EDCF) generated by $A$ and (i) follows. Suppose that (iii) holds. Then $\mathcal{A}$ generates an exponentially bounded $(n+1)$-times integrated semigroup $\left(S_{n+1}(t)\right)_{t \geq 0}$. Define

$$
\mathcal{G}(\varphi):=(-1)^{n+1} \int_{0}^{\infty} \varphi^{(n+1)}(t) S_{n+1}(t) d t, \varphi \in \mathcal{D} .
$$

Then $\mathcal{G}$ is a (DSG) in $E^{2}$ generated by $\mathcal{A}$ (cf. [8, 17]). Let us prove that $\mathcal{G}$ is an (EDSG). Suppose $\left\|S_{n+1}(t)\right\| \leq M e^{\omega t}, \omega \in \mathbb{R}$. Then, for every $a>0$ and $\varphi \in \mathcal{D}$, we have

$$
\begin{aligned}
\left\|\left\langle e^{-(\omega+a) t} \mathcal{G}, \varphi\right\rangle\right\| & \leq M \int_{0}^{\infty} e^{\omega t}\left|\left(e^{-(\omega+a) \cdot} \varphi\right)^{(n)}(t)\right| d t \\
& \leq M 2^{n} \int_{0}^{\infty} e^{\omega t} \sum_{i=0}^{n}|\omega+a|^{n-i} e^{-(\omega+a) t}\left|\varphi^{(i)}(t)\right| d t \\
& \leq M_{1} \int_{0}^{\infty} e^{-a t} \sum_{i=0}^{n}\left|\varphi^{(i)}(t)\right| d t \leq \frac{M_{1}}{a} \sum_{i=0}^{n}\|\varphi\|_{0, i} .
\end{aligned}
$$

It follows: $e^{-(\omega+a) t} \mathcal{G} \in \mathcal{S}^{\prime}\left(L\left(E^{2}\right)\right), \mathcal{G}$ is an (EDSG) and (ii) is valid. If (ii) holds, then there exists $\omega>0$ such that $\{\lambda \in \mathbb{C}: \operatorname{Re} \lambda>\omega\} \subset \rho(\mathcal{A})$ and that $\|R(\cdot: \mathcal{A})\|$ 
is polynomially bounded on $\{\lambda \in \mathbb{C}: \operatorname{Re} \lambda>\omega\}$. Therefore, $\Pi_{\omega}=\left\{\lambda^{2}: \lambda \in \mathbb{C}\right.$, $\operatorname{Re} \lambda>\omega\}$ is contained in $\rho(A)$ and $\|R(\cdot: A)\|$ is polynomially bounded on $\Pi_{\omega}$. So (iv) holds. Assume that (iv) is true. Then $\{\lambda \in \mathbb{C}: \operatorname{Re} \lambda>\omega\} \subset \rho(\mathcal{A})$ and $\|R(\cdot: \mathcal{A})\|$ is polynomially bounded on $\{\lambda \in \mathbb{C}: \operatorname{Re} \lambda>\omega\}$. Applying [19, Theorem 1.12], we have that $\mathcal{A}$ generates an exponentially bounded $(n+1)$-times integrated semigroup for some $n \in \mathbb{N}$ and Proposition 1.3 implies (iii).

Let $A$ be a densely defined operator and let $A$ be the generator of an exponentially bounded $\alpha$-times integrated cosine function for some $\alpha \geq 0$. By [21, Theorem 3.3], the adjoint $A^{*}$ of $A$ is the generator of an exponentially bounded $(\alpha+1)$-times integrated cosine function. Then Theorem 4.4 immediately implies:

Proposition 4.5. Let $A$ be a densely defined operator. If $A$ is the generator of an (EDCF) in $E$, then $A^{*}$ is the generator of an (EDCF) in $E^{*}$.

Let $\alpha \geq 0$. Put $\lceil\alpha\rceil:=\inf \left\{n \in \mathbb{N}_{0}: \alpha \leq n\right\}$.

The next theorem is a perturbation result for generators of $\alpha$-times integrated cosine functions.

Theorem 4.6.([21, Theorem 3.1]) Let $A$ be the generator of an exponentially bounded $2 \alpha$-times integrated cosine function $\left(C_{2 \alpha}(t)\right)_{t \geq 0}$ for some $\alpha \geq 0$. If $B \in L(E)$ and $R(B) \subset D\left(A^{\lceil\alpha\rceil}\right)$, then $A+B$ is the generator of an exponentially bounded $2 \alpha$-times integrated cosine function $\left(C_{2 \alpha}^{B}(t)\right)_{t \geq 0}$.

Note that the assumption $R(B) \subset D\left(A^{\lceil\alpha\rceil}\right)$ is not valid for $\alpha>0$ and $B=a I$, $a \in \mathbb{C} \backslash\{0\}$. So the next question arises: if $A$ is the generator of an exponentially bounded $\alpha$-times integrated cosine function for some $\alpha>0$, does the same hold for $A-a I$ ? We give here a partial answer whose explanation is based on a quite simple argument.

Lemma 4.7. Suppose $A$ is the generator of an exponentially bounded $\alpha$-times integrated cosine function $\left(C_{\alpha}(t)\right)_{t \geq 0}$ for some $\alpha>0$. Then, for all $z \in \mathbb{C}$ and $\beta>\alpha+1, A+z I$ is the generator of an exponentially bounded $\beta$-times integrated cosine function.

Proof. Let $\lambda^{\alpha}=e^{\alpha(\ln |\lambda|+i \arg \lambda)}(\lambda \in \mathbb{C} \backslash\{0\})$ and let $\omega>0$ satisfy

$$
\lambda^{\alpha} R\left(\lambda^{2}: A\right) x=\lambda \int_{0}^{\infty} e^{-\lambda t} C_{\alpha}(t) x d t, x \in E, \operatorname{Re} \lambda>\omega .
$$

Thus, there exists $M>0$ such that $\left\|\lambda^{\alpha-1} R\left(\lambda^{2}: A\right)\right\| \leq M, \operatorname{Re} \lambda>\omega+1$. It implies

$$
\left\|\lambda^{\alpha-1} R\left(\lambda^{2}: A+z I\right)\right\|=\left\|\lambda^{\alpha-1} R\left(\lambda^{2}-z: A\right)\right\| \leq|\lambda|^{\alpha-1} \frac{M}{\left|\lambda^{2}-z\right|^{\frac{\alpha-1}{2}}} \leq M_{1},
$$


for all $\lambda \in \mathbb{C}$ such that $\operatorname{Re} \lambda$ is sufficiently large and a suitable constant $M_{1}>0$. An application of [19, Theorem 1.12] ends the proof.

Corollary 4.8. If $A$ is the generator of an (EDCF) then for all $z \in \mathbb{C}$ the operator $A+z I$ is also the generator of an (EDCF).

Proof. Since $A$ generates an (EDCF), Theorem 4.4 implies that there exists $n \in \mathbb{N}$ such that $A$ generates an exponentially bounded $n$-times integrated cosine function. Then Lemma 4.7 implies that the operator $A+z I$ generates an exponentially bounded $(n+2)$-times integrated cosine function. One can apply again Theorem 4.4 to obtain that $A+z I$ generates an (EDCF).

Proposition 4.9. Suppose that $A$ and $-A$ generate exponential distribution semigroups. Then $A^{2}$ is the generator of an (EDCF).

Proof. Since $A$ generates an (EDSG), one can repeat literally the arguments given in the part (ii) $\Rightarrow$ (i) of the proof of Theorem 4.4 to conclude that there exists $n_{1} \in \mathbb{N}$ such that $A$ generates an exponentially bounded $n_{1}$-times integrated semigroup; see also [8, Theorem 4.9]. Similarly, there exists $n_{2} \in \mathbb{N}$ such that $-A$ generates an exponentially bounded $n_{2}$-times integrated semigroup. Put $n=\max \left(n_{1}, n_{2}\right)$. Then $A$ and $-A$ generate exponentially bounded $n$-times integrated semigroups and by [2, Theorem 5.1], the operator $A^{2}$ generates an exponentially bounded $n$-times integrated cosine function. An application of Theorem 4.4 ends the proof.

Let us turn now to an elementary property of a (DCF).

Proposition 4.10. Let $G$ be a (DCF). Then $G(\varphi) G(\psi)=G(\psi) G(\varphi), \varphi, \psi \in \mathcal{D}$.

Proof. Let $A$ be the generator of $G$. Then there exist $n \in \mathbb{N}$ and $\tau \in(0, \infty)$ such that $A$ is the generator of an $n$-times integrated cosine function $\left(C_{n}(t)\right)_{t \in[0, \tau)}$ satisfying

$$
G(\varphi) x=(-1)^{n} \int_{0}^{\infty} \varphi^{(n)}(t) C_{n}(t) x d t, x \in E, \varphi \in \mathcal{D}_{(-\infty, \tau)} .
$$

From the proof of Theorem 3.2 (we use the same terminology), with

$$
G_{1}(\varphi) x=(-1)^{2^{k}(n+1)} \int_{0}^{\infty} \varphi^{\left(2^{k}(n+1)\right)}(t) S_{2^{k}(n+1)}^{1}(t) x d t, x \in E, \varphi \in \mathcal{D}_{\left(-\infty, 2^{k} \tau\right)},
$$


is given a (DCF) generated by $A$, and Corollary 3.11 implies that

$$
G(\varphi) x=(-1)^{2^{k}(n+1)} \int_{0}^{\infty} \varphi^{\left(2^{k}(n+1)\right)}(t) S_{2^{k}(n+1)}^{1}(t) x d t, x \in E, \varphi \in \mathcal{D}_{\left(-\infty, 2^{k} \tau\right)}
$$

Moreover, by the proof of Theorem 3.2, it follows that $\left(S_{2^{k}(n+1)}^{1}(t)\right)_{t \in\left[0,2^{k} \tau\right)}$ is a $\left(2^{k}(n+1)\right)$-times integrated cosine function. Then Proposition 1.5 implies

$$
S_{2^{k}(n+1)}^{1}(t) S_{2^{k}(n+1)}^{1}(s)=S_{2^{k}(n+1)}^{1}(s) S_{2^{k}(n+1)}^{1}(t), 0 \leq t, s<2^{k} \tau .
$$

Suppose $\varphi, \psi \in \mathcal{D}_{\left(-\infty, 2^{k} \tau\right)}$ for some $k \in \mathbb{N}$. Then the computation

$$
\begin{aligned}
G(\varphi) G(\psi) x & =\int_{0}^{\infty} \varphi^{\left(2^{k}(n+1)\right)}(t) S_{2^{k}(n+1)}^{1}(t) \int_{0}^{\infty} \psi^{\left(2^{k}(n+1)\right)}(s) S_{2^{k}(n+1)}^{1}(s) x d s d t \\
& =\int_{0}^{\infty} \int_{0}^{\infty} \varphi^{\left(2^{k}(n+1)\right)}(t) \psi^{\left(2^{k}(n+1)\right)}(s) S_{2^{k}(n+1)}^{1}(t) S_{2^{k}(n+1)}^{1}(s) x d s d t \\
& =\int_{0}^{\infty} \psi^{2^{k}(n+1)}(s) S_{2^{k}(n+1)}^{1}(s) \int_{0}^{\infty} \varphi^{\left.2^{k}(n+1)\right)}(t) S_{2^{k}(n+1)}^{1}(t) x d t d s \\
& =G(\psi) G(\varphi) x, x \in E, \text { ends the proof. }
\end{aligned}
$$

Question. Does the same hold if $G$ is a pre-(DCF)?

\section{Dense Distribution Cosine Functions}

In this section we focus our attention on dense distribution cosine functions and their generators. Firstly, we will prove

Proposition 5.1. Let $G$ be a (DCF). Then for all $\left(\begin{array}{l}x \\ y\end{array}\right) \in \mathcal{R}(\mathcal{G})$ there exists a function $u \in C^{1}([0, \infty): E)$ satisfying $u(0)=x, u^{\prime}(0)=y$ and

$$
G(\psi) x+G^{-1}(\psi) y=\int_{0}^{\infty} \psi(t) u(t) d t, \psi \in \mathcal{D} .
$$

Proof. It is clear that $\mathcal{G}$ is a (DSG) in $E^{2}$. Since $\mathcal{G}$ satisfies $\left(d_{4}\right)$ (cf. Lemma 1.9) we have that for all $\left(\begin{array}{l}x \\ y\end{array}\right) \in \mathcal{R}(\mathcal{G})$ there exist two functions $u, v \in C([0, \infty): E)$ 
such that $u(0)=x, v(0)=y$ and

$$
\begin{gathered}
G(\psi) x+G^{-1}(\psi) y=\int_{0}^{\infty} \psi(t) u(t) d t, \\
\left(G^{\prime}-\delta\right)(\psi) x+G(\psi) y=\int_{0}^{\infty} \psi(t) v(t) d t, \psi \in \mathcal{D} .
\end{gathered}
$$

With $y=0$ and $x \in E$, integration by parts implies

$$
\int_{0}^{\infty} \varphi^{\prime}(t)\left(u(t)-\int_{0}^{t} v(s) d s\right) d t=G\left(\varphi^{\prime}\right) x+G^{\prime}(\varphi) x=0, \varphi \in \mathcal{D}_{0} .
$$

Then one obtains $u(t)=x+\int_{0}^{t} v(s) d s, t \geq 0$ (cf. for instance [5, Lemma 8.1.1]), and the function $u$ has the desired properties.

Proposition 5.2. Let $G$ be a (DCF) generated by $A$. Then for all $x, y \in D_{\infty}(A)$ there exists a function $u \in C^{1}([0, \infty): D(A))$ satisfying $u(0)=x, u^{\prime}(0)=y$ and

$$
G(\varphi) x+G^{-1}(\varphi) y=\int_{0}^{\infty} \varphi(t) u(t) d t, \varphi \in \mathcal{D}_{0}
$$

Proof. Applying [17, Corollary 3.9], we obtain that for all $\left(\begin{array}{l}x \\ y\end{array}\right) \in D_{\infty}(\mathcal{A})=$ $D_{\infty}(A) \times D_{\infty}(A)$ there exist two functions $u, v \in C([0, \infty): E)$ such that $u(0)=$ $x, v(0)=y$ and

$\mathcal{G}(\varphi)\left(\begin{array}{l}x \\ y\end{array}\right)=\int_{0}^{\infty} \varphi(t)\left(\begin{array}{l}u(t) \\ v(t)\end{array}\right) d t$ for all $\varphi \in \mathcal{D}_{0}$. It implies

$G(\varphi) x+G^{-1}(\varphi) y=\int_{0}^{\infty} \varphi(t) u(t) d t$ and $\left(G^{\prime}-\delta\right)(\varphi) x+G(\varphi) y=\int_{0}^{\infty} \varphi(t) v(t) d t, \varphi \in \mathcal{D}_{0}$.

This leads to the desired result by an argument given in the proof of Proposition 5.1.

Theorem 5.3. Let $G$ be a (DCF) generated by $A$. Then $\overline{D(A)}=E$ if and only if $G$ is dense.

Proof. Assume $G$ is dense. Then Proposition 2.7(i) implies $\overline{D(A)}=E$. Conversely, suppose that $\overline{D(A)}=E$. Since $\rho(A) \neq \emptyset$, we have $\overline{D_{\infty}(A)}=E$ (cf. 
[1] and [10]). So it is enough to show $D_{\infty}(A) \subset \overline{\mathcal{R}(G)}$. Let $x \in D_{\infty}(A)$. By Proposition 5.2, we obtain that there exists a function $u \in C^{1}([0, \infty): E)$ satisfying $u(0)=x, u^{\prime}(0)=0$ and

$$
G(\varphi) x=\int_{0}^{\infty} \varphi(t) u(t) d t, \varphi \in \mathcal{D}_{0} .
$$

Choose $\rho \in \mathcal{D}_{[0,1]}$ with $\int_{\mathbb{R}} \rho(t) d t=1$ and put $\rho_{n}(t)=\rho(n t), t \in \mathbb{R}, n \in \mathbb{N}$. We obtain

$x=\lim _{n \rightarrow \infty} G\left(\rho_{n}\right) x \in \overline{\mathcal{R}(G)}$ and it ends the proof.

Remark. Let $A$ be the generator of a (DCF) $G$. Then one also has: $G$ is dense if and only if $\mathcal{G}$ is an $L$-distribution semigroup in $E^{2}$.

Proof. If $G$ is dense then $\overline{D(\mathcal{A})}=E^{2}$. An employment of [9, Proposition 3.11] gives that $\mathcal{G}$ is a dense (DSG) in $E^{2}$. If $\mathcal{G}$ is an (L-DSG) in $E^{2}$, then its generator $\mathcal{A}$ is densely defined and hence $\overline{D(A)}=E$.

\section{EXAMPLES}

In the preceding sections we saw that generators of distribution cosine functions can be characterized in terms of estimates for their resolvents; we recall that if $A$ generates an (EDCF) then the spectrum $\sigma(A)$ of $A$ must be contained in the parabola $\left\{x+i y: x \leq \omega^{2}-\frac{y^{2}}{4 \omega^{2}}\right\}$ for some $\omega>0$.

Example 6.1. Let $E:=\left\{f \in \bigcap_{k \in \mathbb{N}_{0}} C^{k}[k, \infty): f(0)=0, \quad\|f\|_{E}:=\sup _{k \in \mathbb{N}_{0}} \sup _{t \geq k}\right.$ $\left.\left|f^{(k)}(t)\right|<\infty\right\}$. This space had appeared in [9]. Consider now the operator

$$
A f:=f^{\prime \prime}, D(A):=\left\{f \in E: f^{\prime}, f^{\prime \prime} \in E\right\} .
$$

Suppose that $A$ generates a (DCF). Then there are constants $\omega, M>0$ and $k \in \mathbb{N}$ such that $\left\{\lambda \in \mathbb{R}: \lambda>\omega^{2}\right\} \subset \rho(A)$ and $\left\|R\left(\lambda^{2}: A\right)\right\| \leq M \lambda^{k}, \lambda>\omega$. Choose

$$
g(x)=\left\{\begin{array}{cc}
2 x & , \quad 0 \leq x \leq \frac{1}{2} \\
-2 x+2 & , \quad \frac{1}{2}<x<1 \\
0 & , \quad x \geq 1 .
\end{array}\right.
$$

Then $\|g\|_{E}=1$ and 


$$
\begin{aligned}
M \lambda^{k+1} & \geq\left\|\lambda R\left(\lambda^{2}: A\right) g\right\|_{E}=\left\|\sinh (\lambda t) *_{0} g\right\|_{E} \\
& \geq \frac{1}{2} \sup _{t \geq 0}\left|\int_{0}^{t}\left(e^{\lambda(t-s)}-e^{-\lambda(t-s)}\right) g(s) d s\right| \\
& \geq \sup _{t \in\left[0, \frac{1}{2}\right]}\left|\int_{0}^{t}\left(e^{\lambda(t-s)}-e^{-\lambda(t-s)}\right) s d s\right| \geq \sup _{t \in\left[0, \frac{1}{2}\right]}\left|\frac{-2 t}{\lambda}+\frac{e^{\lambda t}-e^{-\lambda t}}{\lambda^{2}}\right| \\
& \geq\left|\frac{-1}{\lambda}+\frac{e^{\frac{\lambda}{2}}-e^{\frac{-\lambda}{2}}}{\lambda^{2}}\right| \geq \frac{e^{\frac{\lambda}{2}}}{\lambda^{2}}-\frac{1}{\lambda}-\frac{e^{\frac{-\lambda}{2}}}{\lambda^{2}}, \lambda>\omega .
\end{aligned}
$$

Thus, $A$ does not generate a (DCF). Note that operator $-d / d x$ with maximal domain in $E$ is the generator of a (DSG) in $E$ (cf. [9, Example 3.5]).

Examples 6.2. Let $E:=L^{p}((0, \infty)), 1 \leq p \leq \infty, m(x):=\left(x+i e^{x}\right)^{2}, x>0$,

$$
(A f)(x):=m(x) f(x), D(A):=\{f \in E: m f \in E\} .
$$

Clearly, $\left\{x+i e^{x}: x>0\right\} \cap \tilde{\Lambda}_{1,1}=\emptyset$. Denote $d:=\operatorname{dist}\left(\left\{ \pm\left(x+i e^{x}\right): x>0\right\}\right.$, $\left.\partial \widetilde{\Lambda}_{1,1}\right)$. Then $d \in(0, \infty), \tilde{\Lambda}_{1,1}^{2} \subset \rho(A)$, and

$$
\|R(\lambda: A)\| \leq \frac{1}{d^{2}}, \lambda \in \tilde{\Lambda}_{1,1}^{2} .
$$

Therefore, $A$ generates a (DCF). Since $\sigma(A)=\left\{\left(x+i e^{x}\right)^{2}: x>0\right\}$, we have

$$
\sigma(A) \cap \Pi_{\omega} \neq \emptyset \text {, for all } \omega>0 \text {. }
$$

Thus, $A$ does not generate an (EDCF). Moreover, one can easily see that $A$ generates a local once integrated cosine function $\left(C_{1}(t)\right)_{t \in[0,1)}$ on $E$ which is given by

$$
\left(C_{1}(t) f\right)(x)=\frac{\sinh \left(\left(x+i e^{x}\right) t\right) \cdot f(x)}{x+i e^{x}}, 0 \leq t<1, x>0, f \in E .
$$

It is clear that $\left(C_{1}(t)\right)_{t \in[0,1)}$ can be extended on $[0,1]$ and $\sup _{t \in[0,1]}\left\|C_{1}(t)\right\| \leq 1$. However, $A$ does not generate a local sine function on $[0, \tau)$ for any $\tau>1$.

Example 6.3. Let us consider now Hardy spaces of holomorphic functions in the upper half-plane. Denote $\mathbb{R}_{+}^{2}=\{z \in \mathbb{C}: \operatorname{Im} z>0\} . H^{p}\left(\mathbb{R}_{+}^{2}\right), 1 \leq p<\infty$, is defined as follows

$$
\begin{aligned}
H^{p}\left(\mathbb{R}_{+}^{2}\right) & :=\left\{F: F \text { is holomorphic on } \mathbb{R}_{+}^{2} \text { and }\|F\|_{H^{p}\left(\mathbb{R}_{+}^{2}\right)}\right. \\
& \left.:=\left(\sup _{y>0} \int_{\mathbb{R}}|F(x+i y)|^{p} d x\right)^{1 / p}<\infty\right\} .
\end{aligned}
$$


Let $B$ be a holomorphic function on $\mathbb{R}_{+}^{2}$ with $B\left(\mathbb{R}_{+}^{2}\right) \subset\left\{x+i y: x \leq \omega^{2}-\frac{y^{2}}{4 \omega^{2}}\right\}$, for some $\omega>0$, and

$$
(A F)(z):=B(z) F(z), \operatorname{Im} z>0, D(A):=\left\{F \in H^{p}\left(\mathbb{R}_{+}^{2}\right): A F \in H^{p}\left(\mathbb{R}_{+}^{2}\right)\right\} .
$$

One can simply verify $\Pi_{\omega+1} \subset \rho(A)$ and $\left\|\lambda R\left(\lambda^{2}: A\right)\right\| \leq \frac{1}{2 \omega+1}|\lambda|, \operatorname{Re} \lambda>\omega+1$. An application of [19, Theorem 1.12] gives that for all $\alpha>2, A$ is the generator of an exponentially bounded $\alpha$-times integrated cosine function. Thus, $A$ generates an (EDCF) in $H^{p}\left(\mathbb{R}_{+}^{2}\right)$. Particularly, one can take

$$
B(z)=\left(\frac{1}{\pi i} \ln \frac{z-1}{z+1}+a\right)^{2}, \operatorname{Im} z>0(a \in \mathbb{C}), \text { or } B(z)=-\ln ^{2} z, \operatorname{Im} z>0,
$$

where $\ln z=\ln |z|+i \arg (z), z \in \mathbb{C} \backslash\{0\}$.

Example 6.4. Let $E$ be an arbitrary Banach space, $P \in L(E)$ and $P^{2}=P$. Define

$$
G(\varphi) x:=\int_{0}^{\infty} \varphi(t) d t P x, \quad x \in E, \varphi \in \mathcal{D} .
$$

Then $G^{-1}(\varphi) x=\int_{0}^{\infty} t \varphi(t) d t P x, x \in E, \varphi \in \mathcal{D}$. Since $P$ is a continuous projection and

$$
\begin{aligned}
\int_{0}^{\infty} t \varphi(t) & d t \int_{0}^{\infty} \psi(s) d s+\int_{0}^{\infty} \varphi(t) d t \int_{0}^{\infty} s \psi(s) d s \\
= & \int_{0}^{\infty} \int_{0}^{\infty}(t+s) \varphi(t) \psi(s) d s d t \\
= & \int_{0}^{\infty} \int_{0}^{u} u \varphi(u-v) \psi(v) d v d u \\
= & \int_{0}^{\infty} u \int_{0}^{u} \varphi(u-v) \psi(v) d v d u \\
= & \int_{0}^{\infty} u\left(\varphi *_{0} \psi\right)(u) d u, \varphi, \psi \in \mathcal{D}
\end{aligned}
$$

$G$ is a pre-(DCF) in $E$. Moreover, $\{x, y\} \subset \operatorname{KernP} \Leftrightarrow\left(\forall \varphi \in \mathcal{D}_{0}\right) G(\varphi) x+$ $G^{-1}(\varphi) y=0$. Note also that $G$ is a pre-(DSG) in $E$ satisfying $\mathcal{N}(G)=K \operatorname{ern} P$.

Many other examples of exponential distribution cosine functions can be derived from [6, Theorem 3.1] and [2, Theorem 6.6]. The main result of [24] (Theorem) can be used for the construction of exponential distribution cosine functions, too. 


\section{ACKNOWLEDGMENT}

I express my sincere thanks to Professors Stevan Pilipovic and Yizhou Zhang for the helpful discussions. I want also to thank the referee for many useful comments.

\section{REFERENCES}

1. W. Arendt, O. El-Mennaoui and V. Keyantuo, Local integrated semigroups: evolution with the jumps of regularity, J. Math. Anal. Appl., 186 (1994), 572-595.

2. W. Arendt and H. Kellermann, Integrated solutions of Volterra integrodifferential equations and applications, Volterra integrodifferential equations in Banach spaces and applications, Proc. Conf., Trento/Italy 1987, Pitman Res. Notes Math. Ser., 190 (1989), 21-51.

3. W. Arendt, C. J. K. Batty, M. Hieber and F. Neubrander, Vector-valued Laplace Transforms and Cauchy Problems, Birkhauser Verlag, 2001.

4. O. El-Mennaoui and V. Keyantuo, Trace theorems for holomorphic semigroups and the second order Cauchy problem, Proc. Amer. Math. Soc., 124 (1996), 1445-1458.

5. H. O. Fattorini, The Cauchy Problem, Addison-Wesley, 1983. MR 84g:34003.

6. V. Keyantuo, The Laplace transform and the ascent method for abstract wave equations, Journal of Differential Equations, 122 ( 1995), 27-47.

7. J. Kisyński, Distribution semigroups and one parameter semigroups, Bulletin of the Polish Academy of Sciences, 50 (2002), 189-216.

8. M. Kostic, $C$-distribution semigroups, Studia Math., to appear.

9. P. C. Kunstmann, Distribution semigroups and abstract Cauchy problems, Trans. Amer. Math. Soc., 351 (1999), 837-856.

10. P. C. Kunstmann, Stationary dense operators and generation of non-dense distribution semigroups, J. Operator Theory, 37 (1997), 111-120.

11. Y.-C. Li and S.-Y. Shaw, On generators of integrated $C$-semigroups and $C$-cosine functions, Semigroup Forum, 47 (1993), 29-35.

12. M. Li, F. Huang and Q. Zheng, Local integrated $C$-semigroups, Studia Math., 145 (2001), 265-280.

13. J. L. Lions, Semi-groups distributions, Portugal. Math., 19 (1960), 141-164.

14. I. V. Melnikova and A. Filinkov, Abstract Cauchy Problems: Three Approaches, Chapman Hall, Boca Raton, London, New York, Washington, 2001.

15. F. Neubrander, Integrated semigroups and their applications to complete second order Cauchy problems, Semigroup Forum, 38 (1989), 233-251.

16. S.-Y. Shaw, Cosine operator functions and Cauchy problems, Conferenze del Seminario Matematica dell'Universitá di Bari, Dipartimento Interuniversitario Di Matematica, 287, ARACNE, Roma, 2002, pp. 1-75. 
17. S. Wang, Quasi-distribution semigroups and integrated semigroups, J. Funct. Anal., 146 (1997), 352-381.

18. S. W. Wang and Z. Huang, Strongly continuous integrated C-cosine operator functions, Studia Math., 126 (1997), 273-289.

19. T.-J. Xiao and J. Liang, The Cauchy Problem for Higher-Order Abstract Differential Equations, Lect. Notes in Math. Vol. 1701, Springer, 1998.

20. T. Xiao and J. Liang, Differential operators and $C$-wellposedness of complete second order abstract Cauchy problems, Pacific J. Math., 186 (1998), 167-191.

21. J. Zhang and Q. Zheng, On $\alpha$-times integrated cosine functions, Mathematica Japonica, 50 (1999), 401-408.

22. Q. Zheng, Integrated cosine functions, Internat. J. Math. \& Sci., 19, (1996), 575-580.

23. Q. Zheng and J. Zhang, Gaussian estimates and regularized groups, Proc. Amer. Math. Soc., 127 (1999), 1089-1096.

24. Q. Zheng, Coercive differential operators and fractionally integrated cosine functions, Taiwanese J. Math., 6 (2002), 59-65.

Marko Kostic

University of Novi Sad,

Bulevar Oslobodjenja 151,

21000 Novi Sad,

Serbia and Montenegro,

E-mail: makimare@neobee.net 